\title{
Probing the Birth of Post-merger Millisecond Magnetars by X-ray and Gamma-ray Emission
}

\author{
Ling-Jun Wang ${ }^{1,2,3}$, Zi-Gao Dai ${ }^{2,3}$, Liang-Duan $\mathrm{Liu}^{2,3}$, and Xue-Feng $\mathrm{Wu}^{4,5}$ \\ ${ }^{1}$ Key Laboratory of Space Astronomy and Technology, National Astronomical \\ Observatories, Chinese Academy of Sciences, Beijing 100012, China \\ ${ }^{2}$ School of Astronomy and Space Science, Nanjing University, Nanjing 210093, China; \\ dzg@nju.edu.cn \\ ${ }^{3}$ Key laboratory of Modern Astronomy and Astrophysics (Nanjing University), Ministry of \\ Education, Nanjing 210093, China \\ ${ }^{4}$ Purple Mountain Observatory, Chinese Academy of Sciences, Nanjing, 210008, China \\ ${ }^{5}$ Joint Center for Particle Nuclear Physics and Cosmology of Purple Mountain \\ Observatory-Nanjing University, Chinese Academy of Sciences, Nanjing 210008, China
}

\begin{abstract}
There is growing evidence that a stable magnetar could be formed from the coalescence of double neutron stars. In previous papers, we investigated the signature of formation of stable millisecond magnetars in radio and optical/ultraviolet bands by assuming that the central rapidly rotating magnetar deposits its rotational energy in the form of a relativistic leptonized wind. We found that the optical transient PTF11agg could be the first evidence for the formation of post-merger millisecond magnetars. To enhance the probability of finding more evidence for the post-merger magnetar formation, it is better to extend the observational channel to other photon energy bands. In this paper we propose to search the signature of post-merger magnetar formation in X-ray and especially gamma-ray bands. We calculate the SSC emission of the reverse shock powered by post-merger millisecond magnetars. We find that the SSC component peaks at $1 \mathrm{GeV}$ in the spectral energy distribution and extends to $\gtrsim 10 \mathrm{TeV}$ for typical parameters. These energy bands are quite suitable for Fermi/LAT and CTA, which, with their current observational sensitivities, can detect the SSC emission powered by post-merger magnetars up to $1 \mathrm{Gpc}$. NuSTAR, sensible in X-ray bands, can detect the formation of post-merger millisecond magnetars at redshift $z \sim 1$. Future improvement in sensitivity of CTA can also probe the birth of post-merger millisecond magnetars at redshift $z \sim 1$. However, because of the $\gamma-\gamma$ collisions, strong high-energy emission is clearly predicted only for ejecta masses lower than $10^{-3} M_{\odot}$.
\end{abstract}


Subject headings: gamma-ray burst: general — radiation mechanisms: nonthermal — stars: neutron

\section{Introduction}

It is generally accepted that short gamma-ray bursts (SGRBs) result from compact binary mergers, i.e. mergers of binary neutron stars (BNSs) or a neutron star (NS) and a black hole (BH) (Paczyński 1986; Eichler et al. 1989; Barthelmy et al. 2005; Fox et al. 2005; Gehrels et al. 2005; Rezzolla et al. 2011). Such a picture of SGRBs can be confirmed by the upcoming next generation of ground-based gravitational wave (GW) detectors (Harry 2010; Somiya 2012; Acernese et al. 2015; Bartos et al. 2013).

The electromagnetic (EM) signals of compact binary mergers (NS-NS or NS-BH) include SGRBs, kilonovae (also known as macronovae; Li \& Paczyński 1998; Kulkarni 2005; Rosswog 2005; Metzger et al. 2010; Roberts et al. 2011; Metzger \& Berger 2012; Berger et al. 2013; Tanvir et al. 2013; Kasen et al. 2015; Lippuner \& Roberts 2015) ${ }^{1}$, radio afterglow (Nakar \& Piran 2011; Metzger \& Berger 2012; Piran et al. 2013; Rosswog et al. 2013) and possible X-ray emission (Palenzuela et al. 2013).

The coalescence of BNSs appeals much attention because of its rich diversity of possible outcome. In one popular scenario, the supramassive remnant of BNS merger collapses into a black hole on a timescale of $\sim 200 \mathrm{~ms}$ (Faber \& Rasio 2012). Hyperaccretion of a torus around the central black hole could serve as the central engine for GRBs (Popham et al. 1999; Narayan et al. 2001; Kohri \& Mineshige 2002; Liu et al. 2007, 2015; Kawanaka et al. 2013; Xue et al. 2013; Song et al. 2016). In addition, with the assumption of delayed fall-back disk accretion, the black hole could also be the culprit for the X-ray bump and rebrightening following some GRBs (Wu et al. 2013; Geng et al. 2013; Yu et al. 2015a).

Dai et al. (2006) and Zhang (2013), on the other hand, put forward the suggestion that the coalescence of BNSs could have another outcome, i.e. a long-lasting or stable rapidly spinning magnetar. It is constantly demonstrated that the magnetars could be responsible for the persistent emission features of non-afterglow origin following a fraction of SGRBs (Dai et al. 2006; Rowlinson et al. 2010, 2013; Dai \& Liu 2012; Wang \& Dai 2013c; Gompertz et al. 2014; Gao et al. 2015) and the statistical properties of X-ray flares from some GRBs (Wang \& Dai 2013a). The formation of a stable magnetar following the merger

\footnotetext{
${ }^{1}$ There are other possibilities for the claimed kilonova associated with GRB130603B (Takami et al. 2014; Kisaka et al. 2015, 2016)
} 
of BNSs is confirmed by recent simulations in numerical relativity (e.g. Giacomazzo \& Perna 2013; Giacomazzo et al. 2015).

To help identify the formation of stable post-merger magnetars, a line of systematic research (Zhang 2013; Gao et al. 2013a; Wang \& Dai 2013c; Yu et al. 2013, 2015b; Metzger \& Piro 2014; Wu et al. 2014; Wang et al. 2015a; Li \& Yu 2016; Siegel \& Ciolfi 2016a,b) on the magnetar-aided EM signals has recently been carried out based on the energy injection scenarios (Dai \& Lu 1998a,b; Zhang \& Mészáros 2001; Dai 2004). One of the main findings of these studies is that the optical/X-ray transients with a GW association could be not associated with SGRBs. In previous papers (Wang \& Dai 2013c; Wang et al. 2015a), under the assumption of Poynting flux of the spinning-down magnetar becoming lepton dominated $\left(e^{+} e^{-}\right.$pairs; Coroniti 1990; Michel 1994; Dai 2004; Yu \& Dai 2007), we studied the radio and optical/ultraviolet emission based on the recently determined opacities of $r$-process material (Barnes \& Kasen 2013; Kasen et al. 2013).

The basic picture of our model is schematized in Figure 1 of Gao et al. (2013a) and Figure 1 of Wang et al. (2015a). The inspiral and final merger dynamically eject a small amount of neutron-rich material with mass $M_{\mathrm{ej}}=10^{-4}-10^{-2} M_{\odot}$ and subrelativistic velocity $v=0.1-$ 0.3c (Rezzolla et al. 2010; Goriely et al. 2011; Bauswein et al. 2013; Hotokezaka et al. 2013; Rosswog et al. 2013). The merger also forms a torus with mass $\sim 10^{-2} M_{\odot}$ if the merger remnant is a black hole (Fernández \& Metzger 2013). By assuming a constant efficiency in converting torus mass into GRB jet energy, Giacomazzo et al. (2013) find that most of the tori have masses $\lesssim 10^{-2} M_{\odot}$. Neutrino emission from the torus will drive a neutron-rich wind. An even larger quantity of mass could be lost from the torus in a longer viscous time due to outflows driven by viscous heating and recombination of free nuclei into $\alpha$-particles (e.g., Metzger et al. 2008, 2009; Lee et al. 2009; Fernández \& Metzger 2013; Metzger \& Fernández 2014; Just et al. 2015). A long-lived magnetar may significantly enhance the mass loss from the torus because of its long-lasting neutrino irradiation. Metzger \& Fernández (2014) found that a fraction of $\sim 60 \%$ of the torus mass, or equivalently $10^{-2} M_{\odot}$ in mass, may be lost from the torus provided that the merger remnant is a stable magnetar. The remaining torus will be accreted in less than $\sim 1$ s onto the (assumed) remnant magnetar to power an SGRB (Zhang \& Dai 2008, 2009, 2010; Giacomazzo et al. 2013). We therefore expect that the torus disappears after the emergence of SGRB. What left around the magnetar after SGRB is the neutron-rich outflow with mass $\lesssim 10^{-2} M_{\odot}$.

The rapidly spinning massive neutron star builds up its magnetic field to the magnetar level via differential rotation and begins to transfer its rotational energy to the ejecta in the form of Poynting flux $L_{\mathrm{sd}}=L_{\mathrm{sd}, 0} /\left(1+t / T_{\mathrm{sd}}\right)^{2}$, where the spin-down luminosity is $L_{\mathrm{sd}, 0}=10^{47} \mathrm{erg} \mathrm{s}^{-1} P_{0,-3}^{-4} B_{p, 14}^{2} R_{6}^{6}$, the spin-down timescale is $T_{\mathrm{sd}}=2 \times 10^{5} \mathrm{~s}_{6}^{-6} B_{p, 14}^{-2} P_{0,-3}^{2}$, 
$P_{0}$ is the initial rotation period of magnetar, $B_{p}$ is the dipole magnetic field of the magnetar, and $R$ is the neutron star radius. Throughout this paper we adopt the usual convention $Q=10^{n} Q_{n}$. The Poynting flux eventually becomes $e^{ \pm}$-dominated and the transition from Poynting-flux-dominated wind to $e^{ \pm}$-dominated wind could be abrupt (Aharonian et al. 2012). The relativistic magnetar wind is braked by the slow ejecta as discussed above and therefore a reverse shock develops. The accelerated ejecta drive into the ambient interstellar medium and a forward shock develops. The ejecta can be treated as a thin shell (Wang et al. 2015a) despite the effects of radioactive heating (Rosswog et al. 2014).

We showed that the optical transient PTF11agg (Cenko et al. 2013) discovered by the wide-field survey Palomar Transient Factory can be nicely interpreted as the reverse shock synchrotron emission of the leptonized relativistic wind powered by a post-merger millisecond magnetar (Wang \& Dai 2013c). In the paper that follows (Wang et al. 2015a), we systematically investigated all three cases that were studied by Gao et al. (2013a). We found that the very early broadband emission at different wavelengths depends on the properties of the $r$-process material and an ionization breakout is expected based on current knowledge of the $r$-process material.

The electrons in the reverse shock are ultra-relativistic so that inverse Compton (IC) would have an influential effect on the radiative process of electrons. The intense flux of UV/X-ray synchrotron photons produced at the reverse shock serves as seed photons for the relativistic $e^{ \pm}$pairs to inverse Compton scatter them to ultrahigh energy (UHE). In Section 3.2 we show that the thermal and synchrotron photons in the reverse shock could influence the high-energy IC radiation, especially during the early time for the highest energy photons. We find that the IC emission during the magnetar spin-down will be completely attenuated if the ejecta mass $\gtrsim 3 \times 10^{-3} M_{\odot}$.

Based on this result, in this paper, we mainly restrict our attention to the IC emission for Case I, i.e., the low ejecta mass case $\left(M_{\mathrm{ej}}<10^{-3} M_{\odot}\right)$ studied by Wang \& Dai (2013c). Our knowledge about the electromagnetic emission and composition of a newborn magnetar wind comes from the observation and modeling of PWNe. So in Section 2 we clarify the theoretical and observational background of PWNe with an aim to justify our treatment that follows, and leave the open questions in Section 5. Section 3 is divided into two subsections. In Section 3.1 we analytically evaluate the effects of IC on synchrotron emission, while in Section 3.2 we analytically estimate the attenuation of IC photons by the low-energy synchrotron and thermal photons. In Section 4 we propose to detect the birth of post-merger millisecond magnetars by gamma-ray observations. It is found that the emission at the energy band of $1 \mathrm{GeV}$ and $100 \mathrm{GeV}$, suitable for Fermi Large Area Telescope (Fermi/LAT, Atwood et al. 2009) and Cherenkov Telescope Array (CTA, Actis et al. 2011) respectively, can be detected 
up to distances $\sim 1 \mathrm{Gpc}(z \approx 0.2)$ with their current detection sensitivities for a typical millisecond magnetar. We conclude our main findings in Section 6.

\section{Theoretical and Observational Background}

Theoretically, several channels may give birth to millisecond magnetars. One channel to form millisecond magnetars is the double neutron star mergers (Dai \& Lu 1998a,b), viz. the case investigated in this paper. The second channel is the collapse of massive stars, whereby the formation of millisecond magnetars manifests themselves by the emergence of superluminous supernovae (SLSNe) (Kasen \& Bildsten 2010; Woosley 2010; Chatzopoulos et al. 2012; Inserra et al. 2013; Nicholl et al. 2014; Papadopoulos et al. 2015; Wang et al. 2015b,c, 2016; Dai et al. 2016) and luminous SNe (Greiner et al. 2015; Wang et al. 2015d). The third channel is the merger of an NS and a white dwarf (WD; Metzger 2012) with an ejecta mass of $0.3-1 M_{\odot}$. In the latter two cases the ejecta are so massive that the high energy emission cannot be observed immediately following the formation of millisecond magnetars.

The fourth possible channel to form a millisecond magnetar is the accretion-induced collapse (AIC) of a WD (Canal \& Schatzman 1976; Ergma \& Tutukov 1976). Radiationhydrodynamics simulations suggest that a total mass of a few times $10^{-3} M_{\odot}$ be ejected during the collapse of the WD to NS (Dessart et al. 2006). Such a mass is high enough to block the gamma rays emanated from a reverse shock during the early spin-down time of the nascent millisecond magnetar. In addition, whether or not the WD will collapse into an NS or undergo a thermonuclear explosion as a Type Ia supernova in an AIC depends on the mass accretion rate, the WD mass, and the WD composition (Nomoto 1982; Nomoto et al. 1984; Nomoto \& Kondo 1991). The collapse rate of a WD into an NS could be very low and the ejecta composition is also quite different from that coming from the compact binary mergers (Fryer et al. 1999). Based on these considerations, we will restrict ourselves to the magnetars formed during the coalescence of BNSs in this paper.

The rotational energy of the magnetar is converted as Poynting flux within the light cylinder, as can be inferred from the large value of the magnetization parameter $\sigma$, i.e. the ratio of magnetic to particle kinetic energy flux of the flow (Michel 1982; Gaensler \& Slane 2006; Hester 2008). However, to reproduce the observational properties of pulsar wind nebulae (PWNe) the magnetar wind must become particle-dominated upstream of the reverse shock, i.e. the termination shock of the synchrotron PWNe (Rees \& Gunn 1974; Kennel \& Coroniti 1984a,b; Begelman \& Li 1992). The transition mechanism from a high $\sigma$ wind to a low $\sigma$ one, known as the $\sigma$-problem, is as yet poorly understood despite circa five-decade investigations (Kargaltsev et al. 2015). 
One idea that has received a great deal of attention in the past decades is the magnetic reconnection triggered by the annihilation of the alternating magnetic fields (striped wind) near the equatorial plane, generated by an obliquely rotating pulsar, between the light cylinder and the termination shock (Michel 1982, 1994; Coroniti 1990; Lyubarsky \& Kirk 2001; Kirk \& Skjæaasen 2003; Lyubarsky 2003, 2005, 2010a,b; Pétri \& Lyubarsky 2007, 2008; Arons 2012; Hoshino \& Lyubarsky 2012). 1D spherical and 2D axisymmetric MHD models (Atoyan \& Aharonian 1996; Volpi et al. 2008; Olmi et al. 2014) persistently require low value of $\sigma$ upstream of the termination shock. Recent 3D simulations of plasma flow (Mizuno et al. 2011; Porth et al. 2014) suggest instead that the value of $\sigma$ upstream of the termination shock need not to be as low as previously thought, alleviating the $\sigma$-problem.

What makes things even more involved is the abrupt acceleration of the cold ultrarelativistic wind at $20-50 R_{\mathrm{LC}}$ (Aharonian et al. 2012) as inferred under the plausible assumption that the very high energy emission from Crab nebula results from the inverse Compton scattering of pulsed X-ray photons. This fact indicates that the pulsar wind becomes leptondominated and $\sigma \ll 1$ far within the termination shock, challenging current theoretical understanding of the pulsar wind magnetization. On the theoretical side, 1D spherically symmetric MHD models found that the distance of the termination shock from the Crab pulsar could be reproduced only if $\sigma \sim 0.003$ upstream of the shock (Kennel \& Coroniti 1984a,b). 2D simulations indicate that $\sigma \sim 0.02$ works well in accounting for the location of the termination shock and the nebular morphology (Bühler \& Blandford 2014). This value is further raised in recent 3D simulations, $\sigma>1$ (Mizuno et al. 2011; Porth et al. 2014).

But the price in achieving this goal is that the dipolar jet is much weaker than observed (Porth et al. 2014). This argues for an efficient dissipation to convert magnetic energy into dipolar jet energy, in accord with the discovery of abrupt acceleration of the cold wind, viz. a small $\sigma$. For the newborn millisecond magnetars considered in this paper, it is expected that $\sigma$ is even smaller because of the strong dipolar magnetic field and rapid spinning. In the striped wind model, the faster the pulsar is spinning, the shorter the stripe wavelength. In addition, the stronger the dipolar magnetic field, the easier the magnetic reconnection to occur. These two aspects make the dissipation of Poynting flux to particle flux more efficient for millisecond magnetars.

If $\sigma$ is low enough, i.e. $\sigma \lesssim 10^{-3}$ (Sironi \& Spitkovsky 2011a), the two-stream Weibel instability (Fried 1959; Weibel 1959; Medvedev \& Loeb 1999) in the plasma will set in and stochastic magnetic field will grow. If, on the other hand, $\sigma$ is high enough, the Weibel instability will be suppressed and the magnetic field in the shocked wind will be dominant due to the compression of the unshocked wind magnetic field (Zhang \& Kobayashi 2005). We cannot exactly evaluate $\sigma$ for a millisecond magnetar based on current theoretical un- 
derstanding of the magnetic dissipation in relativistic wind. As we argued above, the value of $\sigma$ could be much small for a millisecond magnetar. On the observational aspect, the electrons accelerated behind the termination shock of the pulsar wind have a power-law distribution with an index $p=2.1-2.8$ (Kargaltsev et al. 2015), as expected from the relativistic collisionless shock acceleration (Achterberg et al. 2001; Sironi \& Spitkovsky 2009, 2011a; Bühler \& Blandford 2014). Furthermore, Wang \& Dai (2013c) found that the optical transient PTF11agg can be nicely interpreted as an unmagnetized reverse shock emission powered by a millisecond magnetar. This seems to indicate that the magnetization parameter of the wind blown out by the newborn millisecond magnetar powering PTF11agg is quite low, i.e. $\sigma \lesssim 10^{-3}$. In summary, we argue that for millisecond magnetar, $\sigma$ is so low that the Weibel instability could grow and the electrons in the reverse shock have a power-law distribution with index $2<p<3$.

The magnetic field in the striped wind cannot completely annihilate into electron pairs because the field lines are poloidal near the axis rather than alternating as they are near the equatorial plane. This seems to pose a concern for our assumption that the wind is dominated by leptons. In fact, however, the pulsar wind is highly anisotropic so that the Poynting flux varies with the polar angle as $\sin ^{2} \theta$ (Michel 1973; Bogovalov 1999; Komissarov 2013; Kargaltsev et al. 2015), i.e. the Poynting flux decreases monotonically from the equatorial plane to the polar axis. Numerical simulations show an even more anisotropic distribution that varies as $\sin ^{4} \theta$ (Tchekhovskoy et al. 2013). Therefore the rotational energy of the pulsar is carried away predominantly along the equatorial plane where the magnetic energy is transferred into electron pairs efficiently and our treatment is justified. ${ }^{2}$

The particle Lorentz factor of the pulsar wind usually takes on the values of $\gamma_{4} \sim$ $10^{4}-10^{7}$ (Kennel \& Coroniti 1984a,b; Atoyan 1999; Michel \& Li 1999; Fang \& Zhang 2010; Bucciantini et al. 2011; Tanaka \& Takahara 2011; Wang \& Dai 2013c). In this paper we calculate the IC emission by taking two typical values of $\gamma_{4}=10^{4}$ and $10^{6}$.

The neutron star is also an astrophysical thermal emitter (Potekhin et al. 2015) whose thermal photons from its surface should in principle be taken into consideration. However, because the newborn neutron star cools so rapidly that when the IC photons from the reverse shock manage to penetrate the ejecta, the thermal photons from the neutron star surface become negligible compared with the synchrotron photons. Consequently we ignore thermal emission from the neutron star surface.

\footnotetext{
${ }^{2}$ In some cases strong dissipation is observed near the rotation axis, where the column of baryonic material is likely to be minimized. However, this dissipation along the rotation axis is usually negligibly small compared to the spin-down power of the pulsar.
} 
However, the thermal radiation comes along with the pairs cannot be ignored. In this paper, to be able to tackle the problem in an analytical way and also due to the uncertainty of modeling the thermal radiation accompanied with electron pairs, we assume that the thermal radiation generated along with the pairs takes the form $L_{\mathrm{th}} \propto L_{\mathrm{sd}}$. This assumption is reasonable because thermalization occurs predominantly near the light cylinder and is independent of the ejecta and reverse shock. Figure 2 of Wang et al. (2015a) shows that the synchrotron luminosity $L_{\mathrm{syn}}$ during the spin-down time of the magnetar is nearly a constant. We therefore assume that

$$
L_{\mathrm{th}}=\eta_{\mathrm{th}} L_{\mathrm{syn}}
$$

when $t<T_{\mathrm{sd}}$. For $t>T_{\mathrm{sd}}$ the rotational energy of the magnetar is exhausted and we assume $L_{\mathrm{th}}=0 .{ }^{3}$ We caution that although $L_{\mathrm{syn}}$ is constant during the spin-down of the magnetar, but $L_{\text {syn }}$ depends not only on $L_{\text {sd }}$ but also on the ejecta mass. As a result, $\eta_{\text {th }}$ is not a universal constant and varies from case to case. In the following calculations, we assume $\eta_{\mathrm{th}}=0.2$.

\section{High-energy radiation by $\mathrm{IC}$}

\subsection{Effects of IC on Synchrotron Emission}

The inverse Compton emission of GRB afterglows has been studied and detected for many GRBs (Panaitescu \& Mészáros 1998; Wei \& Lu 1998; Panaitescu \& Kumar 2000; Wang et al. 2001a,b; Harrison et al. 2001; Sari \& Esin 2001; Nakar et al. 2009; Wang et al. 2010; Liu et al. 2013; Wang \& Dai 2013b). It is expected that the IC component of the reverse shock emission in our model is prominent because of the ultrarelativistic nature of the shocked electrons. The IC scattering will boost the cooling of radiative electrons so that the total power of one relativistic electron is

$$
P\left(\gamma_{e}\right)=P_{\text {syn }}\left(\gamma_{e}\right)(1+Y)
$$

where

$$
P_{\mathrm{syn}}\left(\gamma_{e}\right)=\frac{4}{3} \sigma_{T} c \gamma^{2} \beta_{e}^{2} \gamma_{e}^{2} \frac{B^{2}}{8 \pi}
$$

\footnotetext{
${ }^{3}$ To make the analytic calculations affordable, we simplify the problem by assuming that the spin-down power shuts off at the spin-down time, as done in the literature (Gao et al. 2013a; Wang \& Dai 2013c; Wang et al. 2015a). In alignment with this simplification, here we assume that $\eta_{\mathrm{th}}=0$ at spin-down time. Nevertheless, this simplification will not loss important physics because the thermal photons only affect the IC photons at the cut-off energy, as shown in Section 3.2.
} 
$Y$ is the Compton $Y$ parameter and other parameters have their usual meanings (Sari et al. 1998). As a result, the electron cooling Lorentz factor is modified as

$$
\gamma_{c}=\frac{6 \pi m_{e} c}{\sigma_{T} \gamma B^{2} t(1+Y)} .
$$

In determining the ejecta dynamics, we have adopted two different equations, i.e., Equations (5) and (6) in Wang \& Dai (2013c) and Wang et al. (2015a), as follows,

$$
L_{0} \min \left(t, T_{\mathrm{sd}}\right)=\left(\gamma-\gamma_{\mathrm{ej}, 0}\right) M_{\mathrm{ej}} c^{2}+2\left(\gamma^{2}-1\right) M_{\mathrm{sw}} c^{2},
$$

from the energy conservation of the whole system, while

$$
\frac{d \gamma}{d t}=\frac{\xi L_{\mathrm{sd}}+L_{\mathrm{rd}}-L_{\mathrm{ej}, e}-\gamma \mathcal{D}\left(\frac{d E_{3}^{\prime}}{d t^{\prime}}+\frac{d E_{\mathrm{e}, \mathrm{int}}^{\prime}}{d t^{\prime}}\right)-\left(\gamma^{2}-1\right) c^{2}\left(\frac{d M_{\mathrm{sw}}}{d t}\right)}{E_{3}^{\prime}+M_{\mathrm{ej}} c^{2}+E_{\mathrm{e}, \mathrm{int}}^{\prime}+2 \gamma M_{\mathrm{sw}} c^{2}},
$$

for the dynamics in the differential way, where $L_{0}=\xi L_{\mathrm{sd}}$, with $\xi$ the fraction of spindown power of the magnetar that is caught by the ejecta, $L_{\text {sd }}$ the spin-down power of the magnetar, $T_{\mathrm{sd}}$ the spin-down timescale, and $\gamma_{\mathrm{ej}, 0}$ the initial Lorentz factor of the ejecta, $L_{\mathrm{rd}}$ the radioactive heating power due to the $r$-process material, $L_{\mathrm{ej}, e}$ the thermal luminosity of the ejecta, $\mathcal{D}$ the Doppler factor, $E_{3}^{\prime}$ and $E_{\mathrm{ej}, \text { int }}^{\prime}$ the respective energy in Region 3 and in ejecta in the comoving frame. The four regions, i.e. Region 1 to 4 , are defined as: unshocked medium (Region 1), shocked medium (Region 2), shocked wind (Region 3), and unshocked magnetar wind (Region 4), see Figure 1 in Wang et al. (2015a).

Equation (5) originates from Equation (1) in Gao et al. (2013a) and the fact that the energy contained in forward shock and reverse shock is comparable (Blandford \& McKee 1976; Wang \& Dai 2013c). Equation (6), on the other hand, is determined by accounting for all energy in different zones (Dai 2004; Yu et al. 2013; Wang et al. 2015a).

In this section we aim to evaluate the effects of IC on synchrotron spectra and light curves in an analytical manner. To this end it is affordable to adopt Equation (5) rather than (6) for the dynamical evolution of the ejecta because Wang et al. (2015a) showed that Equation (5) is quantitatively equivalent to Equation (6).

As shown in Wang \& Dai (2013c), to analytically calculate the ejecta dynamics and the reverse shock light curves, several timescales have been defined (see Figures 1 and 2 in Wang \& Dai 2013c, for reference.): the transition times between Newtonian dynamics and relativistic dynamics $T_{N 1}$ and $T_{N 2}$, the ejecta deceleration time $T_{\mathrm{dec}}$, the spin-down time of the magnetar $T_{\text {sd }}$, and the time, $T_{\text {ct }}$, i.e. the time at which electron cooling factor $\gamma_{c}$ begins to deviate from unity. The reason for defining $T_{\mathrm{ct}}$ is that the electrons in the reverse shock 
cool so efficiently at beginning that $\gamma_{c}=1$ before $T_{\text {ct }}$. There are two other timescales that are important for the calculation of reverse shock light curves: $T_{a c}$ and $T_{m c}$, the respective crossing time of cooling frequency $\nu_{c}$ with synchrotron self-absorption frequency $\nu_{a}$ and the typical frequency $\nu_{m}$.

IC scattering affects the cooling of electrons so we shall expect that the timescales $T_{\text {ct }}$, $T_{a c}$ and $T_{m c}$ would be modified when IC is taken into account. The Compton $Y$ parameter is given by (Sari \& Esin 2001)

$$
Y=\frac{1}{2}\left(\sqrt{1+4 \eta \frac{\epsilon_{e}}{\bar{\epsilon}_{B}}}-1\right),
$$

which can be simplified as

$$
Y= \begin{cases}\frac{\eta \epsilon_{e}}{\bar{\epsilon}_{B}}, & \frac{\eta \epsilon_{e}}{\bar{\epsilon}_{B}} \ll 1, \\ \left(\frac{\eta \epsilon_{e}}{\bar{\epsilon}_{B}}\right)^{1 / 2}, & \frac{\eta \epsilon_{e}}{\bar{\epsilon}_{B}} \gg 1,\end{cases}
$$

in the limiting cases. Here $\eta$ is the fraction of the electron energy that is radiated away by synchrotron and IC emission. Compared with Sari \& Esin (2001), here we introduce a new parameter

$$
\bar{\epsilon}_{B}= \begin{cases}\epsilon_{B}\left(1+\eta_{\mathrm{th}}\right), & t<T_{\mathrm{sd}} \\ \epsilon_{B}, & t>T_{\mathrm{sd}}\end{cases}
$$

according to the discussion given in Section 2. $\epsilon_{e}$ and $\epsilon_{B}$ are the fraction of total energy going into electrons and magnetic field, respectively. In the following analytical calculations, we usually take the approximation $\bar{\epsilon}_{B} \approx \epsilon_{B}$.

Before $T_{m c}$, i.e. the transition time between fast cooling and slow cooling, the electrons in the reverse shock are in the fast cooling regime so that $\eta=1$. As determined by Wang \& Dai (2013c), $\epsilon_{B} \simeq 0.1$ so that $\epsilon_{e} / \epsilon_{B} \simeq 9 \gg 1$. By Equation (8) and the definition of $T_{\mathrm{ct}}$ (Wang \& Dai 2013c)

$$
T_{\mathrm{ct}}=\frac{3 \pi m_{e} c}{\sigma_{T} \gamma B_{3}^{2}(1+Y)}
$$

we find

$$
T_{\mathrm{ct}}=7.3 \times 10^{-2} \text { days } L_{0,47}^{-2 / 3} M_{\mathrm{ej},-4}^{5 / 6} \epsilon_{e}^{1 / 12} \epsilon_{B,-1}^{1 / 12} .
$$

In this approximation, i.e. $Y \gg 1$, the other two timescales can also be determined

$$
\begin{gathered}
T_{a c}=0.141 \text { days } L_{0,47}^{-(8 p+25) / 2(6 p+19)} M_{\mathrm{ej},-4}^{(5 p+16) /(6 p+19)} \epsilon_{B,-1}^{(p+2) / 2(6 p+19)} \epsilon_{e}^{(p+3) / 2(6 p+19)} \gamma_{4,4}^{-1 /(6 p+19)}, \\
T_{m c}=0.144 \text { days } L_{0,47}^{-5 / 7} M_{\mathrm{ej},-4}^{6 / 7} \epsilon_{B,-1}^{1 / 14} \epsilon_{e}^{3 / 14} \gamma_{4,4}^{1 / 7}
\end{gathered}
$$


The above numerical values are evaluated for $p=2.2$, a value preferred in Wang \& Dai (2013c). Throughout this paper we evaluate all reverse shock parameters at this value of $p$.

Before $T_{m c}$ the effect of IC on the cooling frequency $\nu_{c}$ is just to modify it by a constant factor so that the temporal scaling indices of $\nu_{c}$ is not amended. But after $T_{m c}$, i.e., when the electrons are in the slow cooling regime, not only the exact value of $\nu_{c}$, but also its temporal scaling indices will be changed if IC dominates the electron cooling.

Wang \& Dai (2013c) showed that $T_{\text {dec }}$ is given by (see also Gao et al. 2013a)

$$
T_{\mathrm{dec}}=0.28 \text { days } L_{0,47}^{-7 / 10} M_{\mathrm{ej},-4}^{4 / 5} n^{-1 / 10},
$$

which is usually larger than $T_{m c}$. Now let us calculate the evolution of $\nu_{c}$ in the time period $T_{m c}<t<T_{\text {dec }}$. Before $T_{m c}$ the electrons are in the fast cooling regime and IC dominates their cooling. So we expect that IC will continue to dominate the cooling of electrons for a while.

In the time period $T_{m c}<t<T_{\mathrm{dec}}$, if IC cooling is ignored, we have $\nu_{c} \propto t^{9}$ and $\nu_{m} \propto t^{-5}$ (Wang \& Dai 2013c) so that $\nu_{c} / \nu_{m} \propto t^{14}$. When IC cooling is taken into account, in the slow IC-dominated stage, we have (Sari \& Esin 2001)

$$
\nu_{c} / \nu_{m}=\left(t / T_{m c}\right)^{14} Y^{-2}
$$

Please note that, upon taking into account IC emission, $T_{m c}$ is no longer the time when $\nu_{c}=\nu_{m}$. Here $T_{m c}$ is similar to the variable $t_{0}^{\mathrm{IC}}$ defined by Sari \& Esin (2001). Substitution of Equation (8) into the above equation in the case of $\eta \epsilon_{e} / \epsilon_{B} \gg 1$ and knowing that

$$
\eta=\left(\gamma_{c} / \gamma_{m}\right)^{-(p-2)}
$$

for slow cooling, we have

$$
Y \simeq\left[\left(\frac{t}{T_{m c}}\right)^{14} \frac{1}{Y^{2}}\right]^{-(p-2) / 4} \sqrt{\frac{\epsilon_{e}}{\epsilon_{B}}}
$$

which gives

$$
Y \simeq\left(\frac{\epsilon_{e}}{\epsilon_{B}}\right)^{1 /(4-p)}\left(\frac{t}{T_{m c}}\right)^{-7(p-2) /(4-p)} .
$$

We finally arrive at for the interval $T_{m c}<t<T_{\text {dec }}$ :

$$
\frac{\nu_{c}}{\nu_{m}}=\left(\frac{t}{T_{m c}}\right)^{28 /(4-p)}\left(\frac{\epsilon_{e}}{\epsilon_{B}}\right)^{-2 /(4-p)} .
$$


IC emission will not dominate the electron cooling when $Y=1$, which occurs at

$$
t^{\mathrm{IC}}=T_{m c}\left(\frac{\epsilon_{e}}{\epsilon_{B}}\right)^{1 / 7(p-2)} \simeq 0.69 \text { days. }
$$

This time is usually later than $T_{\mathrm{dec}}$. Consequently, IC emission usually dominates the electron cooling in the entire stage $T_{m c}<t<T_{\mathrm{dec}}$. The IC emission will gradually become less dominant for electron cooling when the temporal scaling indices of $\nu_{c} / \nu_{m}$ is positive. However, inspection of Table 1 of Wang \& Dai (2013c) indicates that the temporal scaling indices of $\nu_{c} / \nu_{m}$ is non-positive when $t>T_{\mathrm{dec}}$. In other words, the IC emission becomes progressively dominant for electron cooling in the stage $t>T_{\mathrm{dec}}$.

The cooling Lorentz factor in the slow IC-dominated regime is given by

$$
\gamma_{c}=\left[\frac{6 \pi m_{e} c}{\sigma_{T} \gamma B^{2} t}\left(\frac{\epsilon_{B}}{\epsilon_{e}}\right)^{1 / 2} \frac{1}{\gamma_{m}^{(p-2) / 2}}\right]^{2 /(4-p)},
$$

from which we can analytically calculate $\nu_{c}$ for $t>T_{\mathrm{dec}}$. The calculated temporal scaling indices of $\nu_{c}$ are given in Table 1. It is worth mentioning that because $p$ is very close to 2 , the scaling indices of $\nu_{c}$ given in Table 1 are actually nearly identical to that given in Table 1 of Wang \& Dai (2013c). As a result, it is usually accurate enough to use Table 1 of Wang \& Dai (2013c) to assess the time evolution of the various synchrotron quantities.

To quantitatively appreciate the effects of IC on the synchrotron emission, in Figure 1 we compare the evolution of synchrotron characteristic frequencies with/without IC taken into account. In this numerical calculation, we adopt Equation (6) to determine the ejecta dynamics. Before $T_{m c}$, IC cooling is to introduce an extra constant factor $\left(\epsilon_{e} / \epsilon_{B}\right)^{1 / 2}$ for $\nu_{c}$. As a result of this factor, the three times $T_{\mathrm{ct}}, T_{a c}$, and $T_{m c}$ are all delayed relative to the value without IC taken into account (Wang \& Dai 2013c), as can be seen from Figure 1. After $T_{m c}$, the temporal scaling indices of $\nu_{c}$ are only slightly changed because $p$ is very close to 2 .

Compared with Equation (5), the adoption of Equation (6) is to increase $\nu_{c}$ when $t>$ $T_{m c}$, as can be seen from Figure 2(b) of Wang et al. (2015a). The consideration of IC is, on the other hand, to decrease $\nu_{c}$ by a nearly same factor. Consequently, we find that the combination of Equation (6) and the consideration of IC cooling results in a cooling frequency that is very close to the one determined in Wang \& Dai (2013c), where we showed that the optical transient PTF11agg can be accounted for by the synchrotron emission of the reverse shock powered by a millisecond magnetar.

The decrease of $\nu_{c}$ caused by IC cooling can be easily calculated. Substitution of $\epsilon_{e} / \epsilon_{B} \simeq$ 9 and $\eta=1$ for fast cooling into Equation (7) gives $Y \simeq 2.5$. During $T_{m c}<t<T_{\text {dec }}, Y$ 
declines slightly, as indicated by Equation (18). But because $p$ is very close to 2, the decline is not significant so that we still have $Y>1$. When $t>T_{\mathrm{dec}}, Y$ begins to increase slowly and actually remains close to $\sim 1.5$. Therefore, IC cooling decreases $\nu_{c}$ by a factor $\sim 7$.

\subsection{Optical depth to $\gamma-\gamma$ collisions}

The photons boosted by IC scattering acquire such a high energy that they will annihilate with the low-energy synchrotron and thermal photons. It is necessary to estimate the optical depth to $\gamma-\gamma$ collisions so that we know under what conditions $\gamma-\gamma$ collisions can be neglected. Figures 5-8 show that the synchrotron photons are usually scattered to a maximum energy of $\sim 10^{13} \mathrm{eV}$. Therefore we set the maximum energy of the IC-scattered photons as $h \nu_{\max }=10^{13} e_{13} \mathrm{eV}$, where $h$ is the Plank constant. The frequency of the softest photons that can annihilate with $h \nu_{\max }$ is given by (e.g., Lithwick \& Sari 2001)

$$
\nu_{\max , a n, \mathrm{dec}}^{\mathrm{I}}=\left(\frac{\gamma_{\mathrm{dec}} m_{e} c^{2}}{h}\right)^{2} \frac{1}{\nu_{\max }}=2.8 \times 10^{14} \mathrm{~Hz} L_{0,47}^{3 / 5} M_{\mathrm{ej},-4}^{-2 / 5} e_{13}^{-1} n^{-1 / 5} .
$$

This frequency is in the optical to UV bands because $e_{13} \lesssim 1$. The above calculation is carried out at time $T_{\text {dec }}$ by setting the Lorentz factor as $\gamma_{\text {dec }}$. This is based on the observation that $T_{\mathrm{dec}} \simeq 0.2$ days at which the spectra of IC emission are calculated below (see Section 4.1 for the reason why we choose this time).

Comparison of $\nu_{\max , a n, \mathrm{dec}}^{\mathrm{I}}$ with Equations (10) and (11) of Wang \& Dai (2013c) shows that $\nu_{\text {max }, a n, \text { dec }}^{\mathrm{I}}$ lies in the range $\nu_{m}<\nu_{\text {max }, a n, \text { dec }}^{\mathrm{I}}<\nu_{c}$. At time $T_{\text {dec }}$ the reverse shock is in the slow cooling regime so that $\nu_{a}<\nu_{m}<\nu_{c}$, and the synchrotron spectrum is $F_{\nu}=$ $F_{\nu, \text { max }}\left(\nu / \nu_{m}\right)^{-(p-1) / 2} \equiv g_{1} \nu^{-\alpha_{1}}$ for a frequency in the range $\nu_{m}<\nu<\nu_{c}$. The synchrotron photon number with frequency above $\nu$ is

$$
N_{>\nu}^{\mathrm{I}}=4 \pi D_{L}^{2} \frac{g_{1}}{h} T_{\mathrm{dec}} \frac{\nu^{-\alpha_{1}}}{\alpha_{1}},
$$

where $\alpha_{1}=(p-1) / 2<1$ if $p<3$.

In the center-of-mass frame of the two colliding photons, the annihilation cross section is approximately $\sigma_{T}$ if their energy is just enough to create electron pair. For higher energy photons the cross section goes to zero as a power law of the photon energy. For a power-law distribution of the seed photons, i.e. $F_{\nu}=g_{1} \nu^{-\alpha_{1}}$, the average $\gamma-\gamma$ collision cross section can be parameterized as $\sigma_{\gamma \gamma}=f\left(\alpha_{1}\right) \sigma_{T}$ with $f\left(\alpha_{1}\right)<1$. In particular $f(1)=11 / 180$ (Svensson 1987; Lithwick \& Sari 2001). ${ }^{4}$ For harder spectrum, i.e. smaller $\alpha_{1}, f\left(\alpha_{1}\right)$ is smaller. The

\footnotetext{
${ }^{4}$ The $\alpha_{1}$ defined in this paper is smaller by 1 than $\alpha$ in Lithwick \& Sari (2001).
} 
synchrotron $\gamma-\gamma$ annihilation optical depth is

$$
\tau_{\mathrm{syn}, \gamma \gamma}^{\mathrm{I}}=\frac{f\left(\alpha_{1}\right) \sigma_{T} N_{>\nu_{\mathrm{max}, a n, \mathrm{dec}}^{\mathrm{I}}}}{4 \pi\left(4 \gamma_{\mathrm{dec}}^{2} c T_{\mathrm{dec}}\right)^{2}}=0.97 \frac{(p-2)^{p-1}}{(p-1)^{p}} n_{-1}^{7(p+1) / 20} e_{13}^{(p-1) / 2} L_{0,47}^{(7-3 p) / 10} \gamma_{4,4}^{p-2} \epsilon_{e}^{p-1} \epsilon_{B,-1}^{(p+1) / 4} M_{\mathrm{ej},-4}^{(p+1) / 5} .
$$

The above numerical value is obtained by setting $\alpha_{1}=1, p=2.2$. The actual value is slightly smaller because $\alpha_{1}<1$ and therefore $f\left(\alpha_{1}\right)<f(1)$.

We see that the optical depth $\tau_{\mathrm{syn}, \gamma \gamma}$ at $T_{\mathrm{dec}}$ is close to unity for typical parameter values. At $t<T_{\text {dec }}$ the synchrotron radiation in the reverse shock is intense enough that the highest energy IC photons are opaque to $\gamma-\gamma$ collision. But in this paper we mainly focus on the IC photons with energy $100 \mathrm{GeV}$ and $1 \mathrm{GeV}$. Equation (24) only weakly depends on $L_{0,47}$, and $\gamma_{4,4}$. The parameter $\epsilon_{e}$ does not concern us because $\epsilon_{e} \simeq 1$. For the $100 \mathrm{GeV}$ IC photons, a simple extrapolation of Equation (24) indicates that an ejecta mass $M_{\mathrm{ej}}=$ $7.5 \times 10^{-3} M_{\odot}$ will make the IC emission attenuated at $T_{\mathrm{dec}}$. However, the ejecta as massive as $M_{\mathrm{ej}}=7.5 \times 10^{-3} M_{\odot}$ are not subject to the above analysis because the above analysis is valid only for case I, i.e. $M_{\mathrm{ej}}<M_{\mathrm{ej}, c}$ or equivalently $T_{\mathrm{sd}}>T_{\mathrm{dec}}$, where $M_{\mathrm{ej}, c}$ is defined as (Gao et al. 2013a)

$$
M_{\mathrm{ej}, c} \sim 10^{-3} M_{\odot} n^{1 / 8} I_{45}^{5 / 4} L_{0,47}^{-3 / 8} P_{0,-3}^{-5 / 2} \xi^{5 / 4}
$$

To find the critical ejecta mass at which the $100 \mathrm{GeV}$ IC photons are completely attenuated by the soft photons, we need to extend the above analysis to Case III, i.e. $M_{\mathrm{ej}}>M_{\mathrm{ej}, c}$ or equivalently $T_{\mathrm{sd}}<T_{\mathrm{dec}}$. In case III $T_{\mathrm{dec}}$ is defined as (Gao et al. 2013a; Wang et al. 2015a)

$$
T_{\mathrm{dec}}=0.9 \text { days } L_{0,48}^{-7 / 3} T_{\mathrm{sd}, 4}^{-7 / 3} M_{\mathrm{ej},-3}^{8 / 3} n^{-1 / 3}
$$

$T_{\text {dec }}$ could be several days as long as $M_{\mathrm{ej}}>10^{-3} M_{\odot}$. We will evaluate the optical depth to $\gamma-\gamma$ collisions at the time $T_{\mathrm{sd}} \sim 1$ days. In this case we have the following softest photons that can annihilate $h \nu_{\max }$

$$
\nu_{\max , a n, \mathrm{sd}}^{\mathrm{III}}=\left(\frac{\gamma_{\mathrm{sd}} m_{e} c^{2}}{h}\right)^{2} \frac{1}{\nu_{\max }}=2.0 \times 10^{14} \mathrm{~Hz}_{0,48}^{2} T_{\mathrm{sd}, 4}^{2} M_{\mathrm{ej},-3}^{-2} e_{13}^{-1} .
$$

At the time $T_{\text {sd }}$ the reverse shock is in the fast cooling regime, viz. $\nu_{c}<\nu_{a}<\nu_{m}$, and inspection of Equations $(28)-(30)$ of (Wang et al. 2015a) indicates that $\nu_{m}<\nu_{\max , a n, \text { sd }}^{\text {III }}$. The synchrotron spectrum is given by $F_{\nu}=F_{\nu, \max }\left(\nu / \nu_{m}\right)^{-p / 2}\left(\nu_{m} / \nu_{c}\right)^{-1 / 2} \equiv g_{2} \nu^{-\alpha_{2}}$ for $\nu>\nu_{m}$, where $\alpha_{2}=p / 2$. The synchrotron photon number with frequency above $\nu$ is

$$
N_{>\nu}^{\mathrm{III}}=4 \pi D_{L}^{2} \frac{g_{2}}{h} T_{\mathrm{sd}} \frac{\nu^{-\alpha_{2}}}{\alpha_{2}} .
$$


The synchrotron $\gamma-\gamma$ annihilation optical depth is

$$
\tau_{\mathrm{syn}, \gamma \gamma}^{\mathrm{III}}=\frac{f\left(\alpha_{2}\right) \sigma_{T} N_{>\nu_{\max , a n, \mathrm{sd}}^{\mathrm{III}}}}{4 \pi\left(4 \gamma_{\mathrm{sd}}^{2} c T_{\mathrm{sd}}\right)^{2}}=\frac{6.2}{p}\left(\frac{p-2}{p-1}\right)^{p-1} e_{13}^{p / 2} \gamma_{4.4}^{p-2} L_{0,48}^{-\frac{11 p}{4}}-\frac{7}{2} T_{\mathrm{sd}, 4}^{-\frac{7 p}{2}-6} \epsilon_{e}^{p-1} \epsilon_{B,-1}^{\frac{p+2}{4}} M_{\mathrm{ej},-3}^{3 p+5} .
$$

The above numerical value is evaluated at $p=2.2$ and $f\left(\alpha_{2}\right)$ is set to $f(1)$ because $p$ is close to 2 and therefore $\alpha_{2}$ is close to 1 . For the $100 \mathrm{GeV}$ IC photons, adopting $L_{0}=10^{47} \mathrm{erg} \mathrm{s}^{-1}$ and $T_{\mathrm{sd}}=10^{5} \mathrm{~s}$, we find $\tau_{\mathrm{syn}, \gamma \gamma}^{\mathrm{III}}=1$ when $M_{\mathrm{ej}}=M_{\mathrm{ej}, c}^{\mathrm{IC}} \simeq 3 \times 10^{-3} M_{\odot}$. Reducing $\epsilon_{B}$ can help raise the critical ejecta mass but its role is limited because $\tau_{\mathrm{syn}, \gamma \gamma}^{\mathrm{III}}$ depends on $M_{\mathrm{ej}}$ very sensitively.

Now we turn to the thermal photons. At $T_{\text {dec }}$ the electrons in the reverse shock are in slow cooling regime so that the energy is radiated dominantly by the electrons with Lorentz factor $\gamma_{c}$. The total synchrotron power is

$$
L_{\mathrm{syn}}=P_{\mathrm{syn}}\left(\gamma_{c}\right) N_{e}
$$

where the total number of injected electrons is

$$
N_{e}=\frac{L_{0} t}{\gamma_{4} m_{e} c^{2}}
$$

The thermal photons in the reverse shock with (observed) temperature $T$ give rise to a thermal luminosity

$$
L_{\mathrm{th}}=4 \pi R^{2} \sigma\left(T / \gamma_{\mathrm{dec}}\right)^{4} \gamma_{\mathrm{dec}}^{2}
$$

Then Equation (1) gives the temperature at time $T_{\text {dec }}$

$$
T=6.1 \times 10^{4} \mathrm{~K} \eta_{\mathrm{th}}^{1 / 4} L_{0,47}^{19 / 40} \gamma_{4,4}^{-1 / 4} M_{\mathrm{ej},-4}^{-2 / 5} n^{-3 / 40} \epsilon_{B,-1}^{-1 / 4},
$$

or equivalently the frequency of the thermal photons

$$
\nu_{\mathrm{th}} \equiv \frac{k_{B} T}{h} \approx 1.3 \times 10^{15} \mathrm{~Hz} \eta_{\mathrm{th}}^{1 / 4} L_{0,47}^{19 / 40} \gamma_{4,4}^{-1 / 4} M_{\mathrm{ej},-4}^{-2 / 5} n^{-3 / 40} \epsilon_{B,-1}^{-1 / 4} .
$$

One sees that this frequency is only weakly dependent on $\eta_{\text {th }}$, which means that a value of $\eta_{\mathrm{th}}=0.1$ is essentially equivalent to $\eta_{\mathrm{th}}=1$. This frequency is usually higher than the minimum frequency (22) to annihilate the hardest IC photons $(10 \mathrm{TeV})$. As a result, approximately all thermal photons, whose number density in the comoving frame is denoted by $n_{\mathrm{th}, a n}^{\prime}$, can annihilate the $10 \mathrm{TeV}$ photons

$$
n_{\mathrm{th}, a n}^{\prime}=16 \pi \zeta(3)\left(\frac{\nu_{\mathrm{th}}^{\prime}}{c}\right)^{3}=16 \pi \zeta(3)\left(\frac{\nu_{\mathrm{th}}}{c \gamma_{\mathrm{dec}}}\right)^{3}
$$


where $\zeta(z)$ is the Riemann zeta function and $\zeta(3)=1.20206$. Here the prime denotes the quantities in the comoving frame. The optical depth for the thermal photons to annihilate the $10 \mathrm{TeV}$ photons is

$$
\tau_{\mathrm{th}, \gamma \gamma}(10 \mathrm{TeV})=n_{\mathrm{th}}^{\prime} s\left(T^{\prime}\right) \sigma_{T} \Delta_{3}^{\prime}=7.1 \times 10^{3} s \eta_{\mathrm{th}}^{3 / 4} L_{0,47}^{1 / 8} \gamma_{4,4}^{-3 / 4} n^{-1 / 8} \epsilon_{B,-1}^{-3 / 4},
$$

where we parameterize the effective annihilation cross section of the thermal photons by $s\left(T^{\prime}\right) \sigma_{T}$ with $s\left(T^{\prime}\right)<1$. The comoving width of Region 3 is given by (Wang \& Dai 2013c)

$$
\begin{aligned}
\Delta_{3}^{\prime} & =\frac{N_{e}}{4 \pi r^{2} n_{3}}, \\
\frac{n_{3}}{n_{4}} & =4 \bar{\gamma}_{3}+3, \\
\bar{\gamma}_{3} & =\frac{\gamma_{4}}{2} \frac{M_{\mathrm{ej}} c^{2}}{L_{0} t} \\
n_{4} & =\frac{L_{0}}{4 \pi r^{2} \gamma_{4}^{2} m_{e} c^{2}} .
\end{aligned}
$$

This thermal annihilation optical depth is large for a reasonably small $\eta_{\text {th }} \simeq 0.1$. Consequently we expect that the $10 \mathrm{TeV}$ photons be attenuated significantly by the thermal photons.

For the $100 \mathrm{GeV}$ and $1 \mathrm{GeV}$ radiation that we focus on in this paper, the softest photons that annihilate the high energy IC photons have a frequency

$$
\nu_{\max , a n, \mathrm{dec}}=2.8 \times 10^{16} \mathrm{~Hz} L_{0,47}^{3 / 5} M_{\mathrm{ej},-4}^{-2 / 5} e_{11}^{-1} n^{-1 / 5},
$$

which is much harder than the thermal photons $\left(\nu_{\mathrm{th}}\right)$. In this case the thermal photons that can annihilate the high energy IC photons is just the photons at the exponential tail of the thermal distribution

$$
n_{\mathrm{th}, a n}^{\prime}=\frac{8 \pi}{c^{3} \gamma_{\mathrm{dec}}^{3}} \nu_{\mathrm{th}} \nu_{\max , a n, \mathrm{dec}}^{2} \exp \left(-\nu_{\max , a n, \mathrm{dec}} / \nu_{\mathrm{th}}\right)
$$

The annihilation optical depth is therefore much small, whatever value $\eta_{\text {th }}$ reasonably takes,

$$
\tau_{\mathrm{th}, \gamma \gamma}(100 \mathrm{GeV}) \lesssim 2.7 \times 10^{-4}
$$

Here we suppress its parameter dependence because the most influential factor appears in the exponent. The optical depth for $1 \mathrm{GeV}$ photons is even smaller.

To summarize the findings in this subsection, at time $T_{\mathrm{dec}}$, the highest energy IC photons $(10 \mathrm{TeV})$ are attenuated by thermal photons. The $100 \mathrm{GeV}$ photons, on which we focus in this paper, on the other hand, are attenuated by synchrotron photons at time $T_{\text {sd }}=1$ days if 
the ejecta are more massive than $3 \times 10^{-3} M_{\odot}$. In short, the $\gamma-\gamma$ collisions have an influence on the light curves but are usually negligible for our purpose to calculate the $1 \mathrm{GeV}$ and $100 \mathrm{GeV}$ for ejecta mass $10^{-4} M_{\odot}$ and $10^{-3} M_{\odot}$. As a result in the following calculations we neglect $\gamma-\gamma$ collisions but mention the caveat where appropriate.

\section{Detect Newborn Magnetars by IC Emission}

\subsection{Inverse Compton spectra and light curves}

In this subsection we will analytically calculate the IC spectra and light curves in order to determine the best observation strategy: observational energy bands and cadence. We assume the Thomson limit in this analytical treatment and refrain the consideration of the Klein-Nishina effect to Section 4.2 where numerical calculations are performed.

When $t<T_{a c}$, we have $\nu_{c}<\nu_{a}<\nu_{m}$, for which case the IC spectrum is given in Appendix A. When $t>T_{a c}$, whatever $\nu_{a}<\nu_{c}<\nu_{m}$ or $\nu_{a}<\nu_{m}<\nu_{c}$, we adopt the IC spectrum calculated by Sari \& Esin (2001). We have discussed the calculation of $\nu_{c}$ and $\gamma_{c}$ in Section 3.1. With these results, we can easily calculate the temporal evolution of IC break frequencies and IC flux density, which are given in Tables 1 and 2, respectively.

Provided that the observational bands lie in the range $\nu>\max \left(\nu_{m m}^{\mathrm{IC}}, \nu_{c c}^{\mathrm{IC}}\right)$, which is usually always true if we aim in detecting $\mathrm{GeV}$ emission, we can read off the evolution of IC flux density directly from Table 2 . We find that the flux density always declines except in the period $T_{m c}<t<T_{\mathrm{dec}}$. This fact can be clearly seen from the numerical calculation results, i.e., Figures 2 and 3. But owing to the fact that $p$ is very close to 2 , the light curves in the time period $T_{m c}<t<T_{\text {dec }}$ are almost flat. The rapid increase in the flux density at the very beginning results from the ejecta becoming progressively transparent for $\gamma$-ray emission. Wang et al. (2015a) showed that the opacity at X-ray and $\gamma$-ray bands is caused by the elastic scattering of photons off free electrons in the shocked wind. Consequently the time for the ejecta becoming transparent for $\gamma$-ray emission is given by Equation (43) in Wang et al. (2015a):

$$
T_{\gamma, \text { thin }}=8.2 \times 10^{-3} \text { days } M_{\mathrm{ej},-4}^{2 / 3} L_{0,47}^{-1 / 3} .
$$

The IC component of the reverse shock emission can be observed if it dominates over synchrotron emission. We therefore need to determine the frequency $\nu^{\mathrm{IC}}$ at which the synchrotron component crosses the IC component.

When $t<T_{a c}$, i.e. for $\nu_{c}<\nu_{a}<\nu_{m}$, Appendix A shows that the IC flux density has a spectral index of $-1 / 2$ for a wide frequency range $\nu_{c a}^{\mathrm{IC}}<\nu<\nu_{m m}^{\mathrm{IC}}$. By assuming that $\nu^{\mathrm{IC}}$ lies 
in the synchrotron spectrum segment with spectral index $-p / 2$ and between the IC break frequencies $\nu_{c a}^{\mathrm{IC}}<\nu<\nu_{m m}^{\mathrm{IC}}$ we have

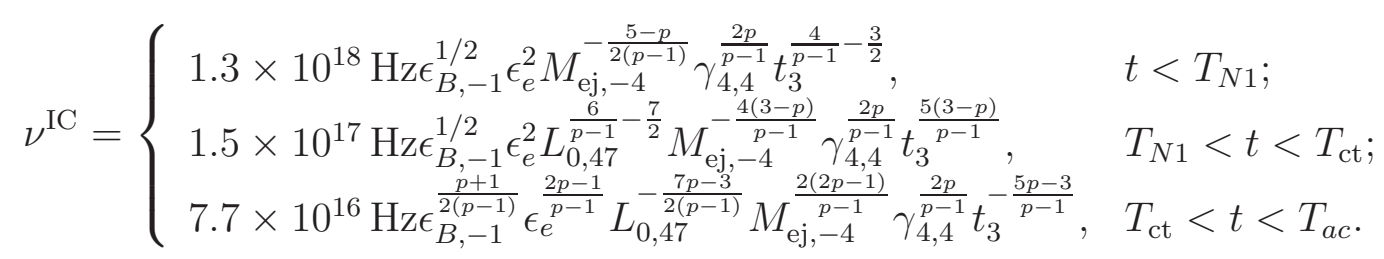

We see that $\nu^{\mathrm{IC}}$ lies in the soft X-ray bands for the typical parameters if $t<T_{a c}$.

When $T_{a c}<t<T_{m c}$, i.e. for $\nu_{a}<\nu_{c}<\nu_{m}$, if $\nu^{\mathrm{IC}}<\nu_{c c}^{\mathrm{IC}}$, we have

$$
\nu^{\mathrm{IC}}=2.5 \times 10^{15} \mathrm{~Hz}_{B,-1}^{-\frac{3(4-p)}{2(3 p+2)}} \epsilon_{e^{\frac{6 p-13}{3 p+2}}}^{\frac{7(22-3 p)}{2(3 p+2)}} M_{0,47}^{-\frac{12(8-p)}{3 p+2}} \gamma_{4,4}^{\frac{6 p}{3 p+2}} t_{4}^{\frac{3(38-5 p)}{3 p+2}}
$$

otherwise we have

$$
\nu^{\mathrm{IC}}=3.2 \times 10^{18} \mathrm{~Hz} \epsilon_{B,-1}^{\frac{p+1}{2(p-1)}} \epsilon_{e^{\frac{2 p-1}{p-1}}}^{-\frac{7 p-3}{2(p-1)}} M_{0,47}^{\frac{2(2 p-1)}{p-1}} \gamma_{4,4}^{\frac{2 p}{p-1}} t_{4}^{-\frac{5 p-3}{p-1}}
$$

if $\nu^{\mathrm{IC}}>\nu_{c c}^{\mathrm{IC}}$.

When $t>T_{m c}$, i.e. for $\nu_{a}<\nu_{m}<\nu_{c}$, if $\nu^{\mathrm{IC}}<\nu_{m m}^{\mathrm{IC}}$, we have

$$
\nu^{\mathrm{IC}}=2.0 \times 10^{16} \mathrm{~Hz} \epsilon_{B,-1}^{\frac{3 p^{2}-10 p+4}{2(p-4)(3 p+2)}} \epsilon^{\frac{2\left(3 p^{2}-8 p-7\right)}{(p-4)(3 p+2)}} L_{0,47}^{-\frac{21 p^{2}-98 p+176}{2(p-4)(3 p+2)}} M_{\mathrm{ej},-4}^{\frac{12\left(p^{2}-5 p+10\right)}{(p-4)(3 p+2)}} \gamma_{4,4}^{\frac{2(p+2)(3 p-11)}{(p-4)(3 p+2)}} t_{4}^{\frac{15 p^{2}-76 p+148}{(4-p)(3 p+2)}}
$$

for $T_{m c}<t<T_{\mathrm{dec}}$

$$
\nu^{\mathrm{IC}}=2.7 \times 10^{18} \mathrm{~Hz} \epsilon_{B,-1}^{\frac{3 p^{2}-10 p+4}{2(p-4)(3 p+2)}} \epsilon^{\frac{2\left(3 p^{2}-8 p-7\right)}{(p-4)(3 p+2)}} L_{0,47}^{-\frac{7 p-34}{2(p-4)(3 p+2)}} n^{\frac{3\left(p^{2}-5 p+10\right)}{2(p-4)(3 p+2)}} \gamma_{4,4}^{\frac{2(p+2)(3 p-11)}{(p-4)(3 p+2)}} t_{4}^{\frac{p+2}{(p-4)(3 p+2)}},
$$

for $T_{\mathrm{dec}}<t<T_{\mathrm{sd}}$

$$
\begin{aligned}
\nu^{\text {IC }}= & 6.9 \times 10^{18} \mathrm{~Hz}_{B,-1}^{\frac{3 p^{2}-10 p+4}{2(p-4)(3 p+2)}} \epsilon_{e^{\frac{2\left(3 p^{2}-8 p-7\right)}{(p-4)(3 p+2)}} L_{0,47}^{-\frac{7 p-34}{2(p-4)(3 p+2)}} n^{\frac{3\left(p^{2}-5 p+10\right)}{2(p-4)(3 p+2)}}} \\
& \times T_{\mathrm{sd}, 5}^{\frac{27 p^{2}-122 p+104}{16(p-4)(3 p+2)}} \gamma_{4,4}^{\frac{2(p+2)(3 p-11)}{(p-4)(3 p+2)}} t_{5}^{-\frac{3\left(9 p^{2}-46 p+24\right)}{16(p-4)(3 p+2)}}
\end{aligned}
$$

for $T_{\mathrm{sd}}<t<T_{N 2} ;$ and

$$
\begin{aligned}
\nu^{\text {IC }}= & 1.2 \times 10^{17} \mathrm{~Hz}_{B,-1}^{\frac{3 p^{2}-10 p+4}{2(p-4)(3 p+2)}} \frac{\frac{2\left(3 p^{2}-8 p-7\right)}{(p-4)(3 p+2)}}{\epsilon^{\frac{3 p^{2}-338 p+1688}{80(p-4)(3 p+2)}}} n^{\frac{117 p^{2}-542 p+872}{80(p-4)(3 p+2)}} \\
& \times T_{\mathrm{sd}, 5}^{\frac{69 p^{2}-334 p+424}{40(p-4)(3 p+2)}} \gamma_{4,4}^{\frac{2(p+2)(3 p-11)}{(p-4)(3 p+2)}} t_{7}^{-\frac{3\left(3 p^{2}-18 p+28\right)}{5(p-4)(3 p+2)}}
\end{aligned}
$$


for $T_{N 2}<t$. On the other hand, if $\nu^{\mathrm{IC}}>\nu_{m m}^{\mathrm{IC}}$, we have

$$
\nu^{\mathrm{IC}}=9.0 \times 10^{13} \mathrm{~Hz} \epsilon_{B,-1}^{\frac{p}{2(p-4)}} \epsilon_{e}^{-\frac{2(p-5)(p-1)}{p-4}} L_{0,47}^{\frac{4 p^{2}-15 p-44}{2(p-4)}} M_{\mathrm{ej},-4}^{-\frac{2\left(p^{2}-3 p-16\right)}{p-4}} \gamma_{4,4}^{-\frac{2(p-5)(p-2)}{p-4}} t_{4}^{\frac{2 p^{2}-5 p-40}{p-4}},
$$

for $T_{m c}<t<T_{\mathrm{dec}}$

$$
\nu^{\mathrm{IC}}=7.9 \times 10^{23} \mathrm{~Hz} \epsilon_{B,-1}^{\frac{p}{2(p-4)}} \epsilon_{e}^{-\frac{2(p-5)(p-1)}{p-4}} L_{0,47}^{\frac{p^{2}-9 p+24}{4(p-4)}} n^{-\frac{p^{2}-3 p-16}{4(p-4)}} \gamma_{4,4}^{-\frac{2(p-5)(p-2)}{p-4}} t_{4}^{-\frac{(p-5) p}{2(p-4)}},
$$

for $T_{\mathrm{dec}}<t<T_{\mathrm{sd}}$

$$
\nu^{\mathrm{IC}}=8.8 \times 10^{24} \mathrm{~Hz} \epsilon_{B,-1}^{\frac{p}{2(p-4)}} \epsilon_{e}^{-\frac{2(p-5)(p-1)}{p-4}} L_{0,47}^{\frac{p^{2}-9 p+24}{4(p-4)}} n^{-\frac{p^{2}-3 p-16}{4(p-4)}} \gamma_{4,4}^{-\frac{2(p-5)(p-2)}{p-4}} T_{\mathrm{sd}, 5}^{-\frac{8 p^{2}-33 p-12}{16(p-4)}} t_{5}^{\frac{7}{16}-\frac{1}{4-p}},
$$

for $T_{\text {sd }}<t<T_{N 2} ;$ and

$\nu^{\text {IC }}=6.4 \times 10^{20} \mathrm{~Hz} \epsilon_{B,-1}^{\frac{p}{2(p-4)}} \epsilon_{e}^{-\frac{2(p-5)(p-1)}{p-4}} L_{0,47}^{\frac{20 p^{2}-195 p+588}{80(p-4)}} n^{-\frac{20 p^{2}-75 p-212}{80(p-4)}} \gamma_{4,4}^{-\frac{2(p-5)(p-2)}{p-4}} T_{\mathrm{sd}, 5}^{-\frac{20 p^{2}-75 p-84}{40(p-4)}} t_{7}^{\frac{5 p-24}{5(p-4)}}$,

for $T_{N 2}<t$. The above expressions are similar to Equation (5.1) in Sari \& Esin (2001).

To determine the maximum energy of IC photons, we need to calculate the maximum Lorentz factor of the electrons in the shocked wind, which is given by

$$
\gamma_{M}=\frac{3}{2} \frac{m_{e} c^{2}}{\sqrt{q^{3} B_{3}(1+Y)}},
$$

if the IC cooling effect is taken into account. Here $B_{3}$ is the magnetic field of Region 3, i.e., the shocked wind.

$$
\gamma_{M}= \begin{cases}2.4 \times 10^{5} M_{\mathrm{ej},-4}^{-1 / 4} \epsilon_{e}^{-1 / 4} t_{3}^{3 / 4}, & t<T_{N 1} \\ 1.9 \times 10^{5} M_{\mathrm{ej}-4}^{-3 / 2} \epsilon_{e}^{-1 / 4} L_{0,47}^{5 / 4} t_{3}^{2}, & T_{N 1}<t<T_{m c} \\ 1.8 \times 10^{7} L_{0,47}^{\frac{5-p}{4(4-p)}} M_{\mathrm{ej},-4}^{-\frac{3 p}{2(4-p)}} \gamma_{4,4}^{-\frac{p-2}{2(4-p)}} \epsilon_{B,-1}^{-\frac{p-2}{4(4-p)}} \epsilon_{e}^{-\frac{p-1}{2(4-p)}} t_{4}^{\frac{3 p+2}{2(4-p)}}, & T_{m c}<t<T_{\mathrm{dec}} \\ 1.8 \times 10^{8} L_{0,47}^{-\frac{p}{16-p)}} n^{-\frac{3 p}{16(4-p)}} \gamma_{4,4}^{-\frac{p-2}{2(4-p)}} \epsilon_{B,-1}^{-\frac{p-2}{4(4-p)}} \epsilon_{e}^{-\frac{p-1}{2(4-p)}} t_{4}^{\frac{8-3 p}{8(4-p)}}, & T_{\mathrm{dec}}<t<T_{\mathrm{sd}}\end{cases}
$$

The maximum energy of the IC photons is given by $\gamma \gamma_{M} m_{e} c^{2}$, which is $\gtrsim 10 \mathrm{TeV}$ for the most likely observational window, i.e., $T_{m c}<t<T_{\text {sd }}$.

With the above calculations, we can now design the observational strategy. To clearly detect the IC component, viz. avoid the contamination from synchrotron component, it is best to observe in $\gamma$-ray bands. From Table 2 and also from Figures 2 and 3, it can be seen that the IC emission declines rapidly when $t>T_{\mathrm{sd}}$. In other words, the IC emission can be detected only in the period $t \lesssim T_{\text {sd }} \sim 1$ days for typical millisecond magnetars with $B_{p}=10^{15} \mathrm{G}$. 


\subsection{Numerical calculations}

To accurately calculate the high-energy spectrum of IC emission of the reverse shock, the Klein-Nishina effect should be taken into account. Unfortunately, the analytical treatment of this effect is complicated and consists of several breaks (Nakar et al. 2009). We therefore take a numerical approach.

The IC volume emissivity in the full precision of Klein-Nishina scattering cross section for an electron distribution $N(\gamma)$ is given by (Blumenthal \& Gould 1970)

$$
j_{\nu}^{\mathrm{IC}}=3 \sigma_{T} \int d \gamma N(\gamma) \int_{\nu_{s, \min }}^{\infty} \frac{\nu}{4 \gamma^{2} \nu_{s}^{2}} g(x, y) F_{\nu_{s}} d \nu_{s},
$$

where $F_{\nu_{s}}$ is the synchrotron seed flux density, $\nu_{s, \min }=\nu / 4 \gamma^{2}, g(x, y)$ is given by

$$
g(x, y)=2 y \ln y+1+y-2 y^{2}+\frac{1}{2} \frac{x^{2} y^{2}}{1+x y}(1-y),
$$

and $x, y$ are defined as

$$
x=\frac{4 \gamma h \nu_{s}}{m_{e} c^{2}}, y=\frac{h \nu}{x\left(\gamma m_{e} c^{2}-h \nu\right)}=\frac{\nu}{4 \gamma^{2} \nu_{s}-x \nu} .
$$

We calculate the IC emission of both the reverse shock and the forward shock numerically, assuming the neutron star mergers locate at a luminosity distance $D_{L}=10^{27} \mathrm{~cm}$. In Figures 2 and 3 we choose the fiducial model (solid lines) as the one with parameters $M_{\mathrm{ej}}=10^{-4} M_{\odot}, L_{\mathrm{sd}}=10^{47} \mathrm{erg} \mathrm{s}^{-1}$, and $T_{\mathrm{sd}}=10^{5} \mathrm{~s}$. To see the effect of varying $M_{\mathrm{ej}}$, we show the light curves for $M_{\mathrm{ej}}=5 \times 10^{-4} M_{\odot}$ as dashed lines. It can be seen that the more massive the ejecta, the easier for it to be observed. The figure also shows that the IC emission from forward shock is unlikely to be observed.

To assess the influence of a different value of $L_{\mathrm{sd}}$, we compare the light curves for $L_{\mathrm{sd}}=5 \times 10^{47} \mathrm{erg} \mathrm{s}^{-1}$ with the fiducial model in Figure 3. What should be noted is that the rotational energy of the magnetars is fixed to be $10^{52} \mathrm{erg}$. As a result, with a spin-down power of $L_{\mathrm{sd}}=5 \times 10^{47} \mathrm{erg} \mathrm{s}^{-1}$, its spin-down timescale is reduced to $T_{\mathrm{sd}}=2 \times 10^{4} \mathrm{~s}$. From Figure 3 we see that the IC emission power is enhanced by the more powerful magnetars, but its duration is shorten significantly. Consequently, only high-cadence observations can reveal the formation of post-merger magnetars with a larger spin-down power.

In the above calculations we set the Lorentz factor of the unshocked wind (Region 4) as $\gamma_{4}=10^{4}$, as determined in the literature (Atoyan 1999; Dai 2004; Wang \& Dai 2013c). The ambient medium density is set as $n=0.1 \mathrm{~cm}^{-3}$ (Berger et al. 2005; Soderberg et al. 2006; 
Berger 2007; Wang \& Dai 2013c). We will first observe the reverse shock emission at time $\sim T_{\gamma \text {,thin }}$ and then the forward shock emission at time $\sim T_{\text {dec }}$ if the forward shock $\gamma$ ray can be observed.

Figure 4 shows the effects of varying $\gamma_{4}$ on the resulting IC light curves. We see that increasing $\gamma_{4}$ will suppress the early IC emission but enhance it later on. This behaviour can be understood as follows. At beginning, the ejecta velocity is low and the electrons' individual energy in the reverse shock is high enough to inverse scatter the photons to high energy. As a result, the IC emission intensity is enhanced because of the lower Lorentz factor of the unshocked wind and hence more numerous electrons in the shocked wind (the wind power $L_{\mathrm{sd}}$ is fixed here). At later time, however, the ejecta become relativistic and the electrons' individual energy in the reverse shock is reduced. In this case, a larger Lorentz factor of the unshocked wind is prone to produce more intense IC emission.

Here we choose the observational bands at energies of $1 \mathrm{GeV}$ and $100 \mathrm{GeV}$. The energy band at $100 \mathrm{GeV}$ is chosen here because this is in the sensitive region for CTA and far below the high-energy cut of the reverse shock IC emission $\gamma \gamma_{M} m_{e} c^{2}$. We choose the energy band at $1 \mathrm{GeV}$ since this is the most sensitive observational band for Fermi/LAT and it is also near the peak energy of the IC spectrum. These points can be easily seen from Figures 5-8.

To appreciate the IC spectrum and its evolution, we show the spectra at times $t=$ 0.2 days and $t=1$ day in Figures 5 (the fiducial model, $M_{\mathrm{ej}}=10^{-4} M_{\odot}, L_{\mathrm{sd}}=10^{47} \mathrm{erg} \mathrm{s}^{-1}$, and $\left.T_{\mathrm{sd}}=10^{5} \mathrm{~s}\right), 6\left(M_{\mathrm{ej}}=5 \times 10^{-4} M_{\odot}\right), 7\left(L_{\mathrm{sd}}=5 \times 10^{47} \mathrm{erg} \mathrm{s}^{-1}\right)$, and $8\left(\gamma_{4}=10^{6}\right)$. From Figure 2 we see that the second peak occurs at $t \simeq 0.2$ days for the fiducial model. This is why we choose to calculate one of the IC spectra at $t=0.2$ days. The reason we do not choose the time when the first peak occurs is that it happens too early and is therefore not easy to be caught by a regular-cadence observation. We choose to calculate the other spectrum at $t=1$ day because $T_{\text {sd }} \simeq 1$ day for a typical magnetar so that its emission is still very strong to be caught by telescopes. These figures also show that the frequencies $\nu^{\mathrm{IC}}$ at which IC emission dominates over synchrotron emission usually occur in the X-ray and $\gamma$-ray bands for typical parameters.

\section{Discussions}

Now our theoretical understanding of the physical processes giving rise to particle acceleration is still incomplete. Fortunately the radiation spectra of PWNe can be used to infer the particle acceleration processes. The radiation spectra of PWNe from radio to a few hundred $\mathrm{MeV}$ is due to synchrotron emission and the high energy part can be interpreted 
as inverse Compton scattering. In this paper we assume that the shock accelerated electrons have a power-law distribution index $2<p<3$, as is the case for most PWNe. However there are some cases that the electrons have a flat (broken) power law slope $N(E) \propto E^{-p}$, where $1<p<2$ (Kargaltsev et al. 2015). Such an electron distribution slope is inconsistent with diffusive shock acceleration (DSA). Theoretical effort suggests that the flat electron distribution could be a result of magnetic reconnection at the termination shock (Lyubarsky 2003; Lyubarsky \& Liverts 2008).

Based on the idea of driven magnetic reconnection, Lyubarsky \& Liverts (2008) managed to show that the particle distribution can be well approximated by a power-law with index -1 and an exponential cutoff. This result is encouraging but the power-law slop is systematically flatter than observational data. 3D simulations (Sironi \& Spitkovsky 2011b; Kargaltsev et al. 2015) found that the electrons accelerated by driven reconnection could have a flat distribution only if the parameter $\xi=\lambda / \sigma r_{L}$, where $\lambda$ is the stripe wavelength, $r_{L}$ the Larmor radius of the electrons upstream of the termination shock, takes on an unrealistic large value. Otherwise the electrons would be in a Maxwellian distribution. Therefore the acceleration of electrons in the relativistic wind is still an unresolved puzzle. It is nev-

ertheless valuable to investigate the emission characteristics of a flat electron distribution in future work.

Another assumption of this paper is the unmagnetized upstream of the termination shock. This assumption is plausible for a newborn millisecond magnetar examined in this paper. We should nevertheless consider the case where the pulsar wind is still magnetized at the termination shock, which is the subject we will examine in an accompanying paper (Liu et al. 2016).

\section{Conclusions}

It seems that we are in an era of deciphering the central engines of SGRBs, given that the next generation of GW detectors are already begining to detect nearby compact binary mergers (Abbott et al. 2016) and that CTA and Fermi/LAT are becoming sensitive enough to catch the high-energy $\gamma$-ray signals.

In previous papers (Wang \& Dai 2013c; Wang et al. 2015a), we calculated the radio and optical/UV radiation of the reverse shock powered by the post-merger millisecond magnetars. We find that the optical transient PTF11agg can be neatly interpreted by the reverse shock synchrotron emission powered by a millisecond magnetar.

In this paper we first evaluate the effects of IC cooling on the synchrotron spectrum and 
light curves, which were ignored in previous papers. The IC cooling only affects the cooling frequency $\nu_{c}$ of the synchrotron emission and the temporal scaling indices of $\nu_{c}$ are changed only slightly. The IC emission enhances the cooling of electrons such that $\nu_{c}$ is reduced relative to that when $\mathrm{IC}$ is ignored. We find, however, that the combination of Equation (6) and IC effect is to result in a cooling frequency that is very close to that calculated in Wang \& Dai (2013c), where the light curves of PTF11agg were calculated.

We then further explore the high-energy emission caused by synchrotron self-Compton (SSC) scattering powered by post-merger millisecond magnetars after analytically estimating the attenuation caused by the $\gamma-\gamma$ collisions between high-energy IC photons and the lowenergy thermal and synchrotron photons. The SSC emission, lasting for $\sim 1$ day, usually dominates synchrotron emission at X-ray bands and extends to the high-energy cutoff at $\gtrsim 10 \mathrm{TeV}$.

We find that for a typical magnetar at a distance $10^{27} \mathrm{~cm}$, the SSC emission can be detected by both Fermi/LAT and CTA during its whole spin-down period $T_{\text {sd }}$. Figures 5-8 show that the high-energy emission can be detected most sensitively and feasibly at the energy bands $1 \mathrm{GeV}$ and $100 \mathrm{GeV}$. NuSTAR (Harrison et al. 2013) can also be helpful in some cases (see Figures 5-8) in identifying the SSC emission in X-ray.

Comparison of Figures 5-8 shows that the SSC component evolves more rapidly if the ejecta are less massive or the spin-down power of the central magnetars is more powerful. The difference of less massive ejecta and a more powerful magnetar can be discerned by noting the fact that the more powerful the central magnetar, the more pronounced the synchrotron component compared to the SSC component, as can been seen by comparing the respective spectra at $t=0.2$ days for the cases $M_{\mathrm{ej}}=10^{-4} M_{\odot}$ (Figure 5 ) and $L_{\mathrm{sd}}=5 \times 10^{47} \mathrm{erg} \mathrm{s}^{-1}$ (Figure 7). Consequently, if the observation cadence on the same sky area is 5 times per day, the first detection of a spectrum from optical to $\mathrm{GeV}$ band (at $t \sim 0.2$ days from the magnetar birth) contains much information about the central magnetars and the ejecta mass. Meanwhile, we find that the high-energy IC emission is only clearly predicted when $M_{\mathrm{ej}} \lesssim 10^{-3} M_{\odot}$.

Fermi/LAT has been in orbit for more than 6 years since its launch. The high-energy reverse shock emission from post-merger millisecond magnetars is, however, very rare so far (Acero et al. 2015). This can be understood in the following ways. If SGRBs are indeed produced by compact binary mergers and a significant fraction of such mergers results in stable magnetars, we should be able to observe the high-energy reverse shock emission. However, SGRBs are usually located at redshifts $z \sim 0.5$ (Berger 2014), which is beyond the detection limit, a few $10^{27} \mathrm{~cm}$, of Fermi/LAT. 
Inspection of the sensitivity of $N u S T A R$, we find that $N u S T A R$ can detect the reverse shock emission powered by post-merger millisecond magnetars up to redshifts $z \gtrsim 1$, i.e. the redshifts for most frequently occurred SGRBs. Future improvement on the sensitivity of CTA will extend the detection limit for high-energy reverse shock emission to $z \gtrsim 1$.

We would like to thank the anonymous referee for very valuable suggestions which have allowed us to improve our manuscript significantly. This work is supported by the National Basic Research Program ("973" Program) of China (grant Nos. 2014CB845800 and 2013CB834900) and the National Natural Science Foundation of China (grant Nos. 11573014, U1331202 and 11322328). X.F.W. is also partially supported by the Youth Innovation Promotion Association (2011231), and the Strategic Priority Research Program "The Emergence of Cosmological Structures" (grant No. XDB09000000) of the Chinese Academy of Sciences.

\section{A. Analytical IC spectrum in the case of $\nu_{c}<\nu_{a}<\nu_{m}$}

In this appendix, we adopt the approximation by Sari \& Esin (2001) to calculate the IC spectrum for use in this paper. To our knowledge, the IC spectra of GRB afterglows for all six cases have been analytically integrated by Gou et al. (2007) and Gao et al. (2013b), besides Sari \& Esin (2001). The relevant cases for this paper are $\nu_{c}<\nu_{a}<\nu_{m}, \nu_{a}<\nu_{c}<\nu_{m}$, and $\nu_{a}<\nu_{m}<\nu_{c}$. For the later two cases we use the result given by Sari \& Esin (2001).

In the case of $\nu_{c}<\nu_{a}<\nu_{m}$, Gao et al. (2013b) considered the heating of low-energy electrons due to synchrotron absorption. For our purpose here, only the high energy IC emission concerns us and so we ignore this low-energy heating. The synchrotron spectrum in the case of $\nu_{c}<\nu_{a}<\nu_{m}$ is given by

$$
F_{\nu}= \begin{cases}F_{\nu, \max }\left(\nu / \nu_{c}\right)^{2}\left(\nu_{c} / \nu_{a}\right)^{3}, & \nu<\nu_{c} \\ F_{\nu, \max }\left(\nu / \nu_{a}\right)^{5 / 2}\left(\nu_{a} / \nu_{c}\right)^{-1 / 2}, & \nu_{c}<\nu<\nu_{a} \\ F_{\nu, \max }\left(\nu / \nu_{c}\right)^{-1 / 2}, & \nu_{a}<\nu<\nu_{m} \\ F_{\nu, \max }\left(\nu / \nu_{m}\right)^{-p / 2}\left(\nu_{m} / \nu_{c}\right)^{-1 / 2}, & \nu_{m}<\nu<\nu_{M}\end{cases}
$$

The IC spectrum is given by the following integration (Sari \& Esin 2001)

$$
f_{\nu}^{\mathrm{IC}}=R \sigma_{T} \int_{\min \left(\gamma_{m}, \gamma_{c}\right)}^{\infty} d \gamma N(\gamma) \int_{0}^{x_{0}} d x f_{\nu_{s}}(x),
$$

where $N(\gamma)$ is the electron number density per unit $\gamma, f_{\nu_{s}}(x)$ is the synchrotron seed photon flux density, $x=\nu / 4 \gamma^{2} \nu_{s}$ with $\nu$ the frequency of emitting IC photons. 
In fast cooling regime, the electron distribution $N(\gamma)$ is given by Equation (2.2) in Sari \& Esin (2001). If $\nu<4 \gamma^{2} \nu_{c} x_{0}$, the inner integral in Equation (A2) gives

$$
I_{1} \simeq \frac{5}{3} F_{\nu, \max } x_{0}\left(\frac{\nu_{c}}{\nu_{a}}\right)^{1 / 2} \frac{\nu}{4 \gamma^{2} \nu_{a} x_{0}} .
$$

If $4 \gamma^{2} \nu_{c} x_{0}<\nu<4 \gamma^{2} \nu_{a} x_{0}$, this same integral is

$$
I_{1}^{\prime} \simeq \frac{4}{3} F_{\nu, \max } x_{0}\left(\frac{\nu_{c}}{\nu_{a}}\right)^{1 / 2} \frac{\nu}{4 \gamma^{2} \nu_{a} x_{0}} .
$$

In the following calculation we take the value given by Equation $(A 4)$ for $\nu<4 \gamma^{2} \nu_{a} x_{0}$. We summarize the inner integral as follows

$$
I= \begin{cases}I_{1} \simeq \frac{4}{3} F_{\nu, \max } x_{0}\left(\frac{\nu_{c}}{\nu_{a}}\right)^{1 / 2} \frac{\nu}{4 \gamma^{2} \nu_{a} x_{0}}, & \nu<4 \gamma^{2} \nu_{a} x_{0} \\ I_{2} \simeq \frac{2}{3} F_{\nu, \max } x_{0}\left(\frac{\nu}{4 \gamma^{2} \nu_{c} x_{0}}\right)^{-1 / 2}, & 4 \gamma^{2} \nu_{a} x_{0}<\nu<4 \gamma^{2} \nu_{m} x_{0} \\ I_{3} \simeq \frac{2}{p+2} F_{\nu, \max } x_{0}\left(\frac{\nu_{c}}{\nu_{m}}\right)^{1 / 2}\left(\frac{\nu}{4 \gamma^{2} \nu_{m} x_{0}}\right)^{-p / 2}, & 4 \gamma^{2} \nu_{m} x_{0}<\nu\end{cases}
$$

For the IC break frequencies we adopt the convenient notation used by Gao et al. (2013b), i.e.,

$$
\nu_{i j}^{\mathrm{IC}} \equiv 4 \gamma_{i}^{2} \nu_{j} x_{0}, \quad i=m, c, j=a, m, c .
$$

As pointed out by Gao et al. (2013b), to get the right result for the double integral $(A 2)$, higher order terms of $(A 5)$ should be taken into account. After integration of Equation $(A 2)$ we finally get

$$
\begin{aligned}
& f_{\nu}^{\mathrm{IC}} \simeq R \sigma_{T} n F_{\nu, \max } x_{0} \\
& \begin{cases}\frac{4}{9}\left(\frac{\nu_{c}}{\nu_{a}}\right)^{1 / 2}\left(\frac{\nu}{\nu_{c a}^{\mathrm{IC}}}\right), & \nu<\nu_{c a}^{\mathrm{IC}} \\
\frac{1}{3}\left(\frac{\nu}{\nu_{c c}^{\mathrm{IC}}}\right)^{-1 / 2}\left[1+\ln \left(\frac{\nu}{\nu_{c a}^{\mathrm{IC}}}\right)\right], & \nu_{c a}^{\mathrm{IC}}<\nu<\nu_{m c}^{\mathrm{IC}} \\
\frac{1}{3}\left(\frac{\nu}{\nu_{c c}^{\mathrm{IC}}}\right)^{-1 / 2}\left[\frac{p+5}{3(p-1)}+\ln \left(\frac{\nu_{m}}{\nu_{a}}\right)\right], & \nu_{m c}^{\mathrm{IC}}<\nu<\nu_{m a}^{\mathrm{IC}} \\
\frac{1}{3}\left(\frac{\nu}{\nu_{c c}^{\mathrm{IC}}}\right)^{-1 / 2}\left[\frac{2(7-p)}{3(p-1)}+\ln \left(\frac{\nu_{m m}^{\mathrm{IC}}}{\nu}\right)\right], & \nu_{m a}^{\mathrm{IC}}<\nu<\nu_{m m}^{\mathrm{IC}} \\
\frac{1}{p+2}\left(\frac{\nu}{\nu_{m m}^{\mathrm{IC}}}\right)^{-p / 2} \frac{\nu_{c}}{\nu_{m}}\left[\frac{6(p+1)}{(p-1)(p+2)}+\ln \left(\frac{\nu}{\nu_{m m}^{\mathrm{IC}}}\right)\right], & \nu_{m m}^{\mathrm{IC}}<\nu .\end{cases}
\end{aligned}
$$

The result given by Gou et al. (2007) is exactly correct if we assume that all higher-order terms in Equation (A5) can be ignored. 


\section{REFERENCES}

Abbott, B. P., Abbott, R., Abbott, T. D., et al. 2016, PhRvL, 116, 061102

Acernese, F., Agathos, M., Agatsuma, K., et al. 2015, CQGra, 32, 024001

Acero, F., Ackermann, M., Ajello, M., et al. 2015, ApJS, 218, 23

Achterberg, A., Gallant, Y. A., Kirk, J. G., \& Guthmann, A. W. 2001, MNRAS, 328, 393

Actis, M., Agnetta, G., Aharonian, F., et al. (The CTA Consortium) 2011, Exp Astron, 32, 193

Aharonian, F. A., Bogovalov, S. V., \& Khangulyan, D. 2012, Nature, 482, 507

Arons, J. 2012, SSRv, 173, 341

Atoyan, A. M. 1999, A\&A, 346, L49

Atoyan, A. M., \& Aharonian, F. A. 1996, MNRAS, 278, 525

Atwood W. B., Abdo, A. A., Ackermann, M., et al. 2009, ApJ, 697, 1071

Barnes, J. \& Kasen, D. 2013, ApJ, 775, 18

Barthelmy, S. D., Chincarini, G., Burrows, D. N., et al. 2005, Nature, 438, 994

Bartos, I., Brady, P., \& Márka, S. 2013, CQGra, 30, 123001

Bauswein, A., Goriely, S., \& Janka, H. T. 2013, ApJ, 773, 78

Begelman, M. C., \& Li, Z. Y. 1992, ApJ, 397, 187

Berger, E. 2007, ApJ, 670, 1254

Berger, E. 2014, ARA\&A, 52, 43

Berger, E., Fong, W., \& Chornock, R. 2013, ApJL, 774, L23

Berger, E., Price, P. A., Cenko, S. B., et al. 2005, Nature, 438, 988

Blandford, R. D., \& McKee, C. 1976, PhFl, 19, 1130

Blumenthal, G. R. \& Gould, R. J. 1970, RvMP, 42, 237

Bogovalov, S. V. 1999, A\&A, 349, 1017 
Bucciantini, N., Arons, J., \& Amato, E. 2011, MNRAS, 410, 381

Bühler, R., \& Blandford, R. 2014, RPPh, 77, 066901

Canal, R., \& Schatzman, E. 1976, A\&A, 46, 229

Cenko, S. B., Kulkarni, S. R., Horesh, A., Corsi, A., \& Fox, D. B. 2013, ApJ, 769, 130

Chatzopoulos, E., Wheeler, J. C., \& Vinko, J. 2012, ApJ, 746, 121

Coroniti, F. V. 1990, ApJ, 349, 538

Dai, Z. G. 2004, ApJ, 606, 1000

Dai, Z. G. \& Liu, R. Y. 2012, ApJ, 759, 58

Dai, Z. G., \& Lu, T. 1998a, A\&A, 333, L87

Dai, Z. G., \& Lu, T. 1998b, PhRvL, 81, 4301

Dai, Z. G., Wang, S. Q., Wang, J. S., Wang, L. J., \& Yu, Y. W. 2016, ApJ, 817, 132

Dai, Z. G., Wang, X. Y., Wu, X. F., \& Zhang, B. 2006, Science, 311, 1127

Dessart, L., Burrows, A., Ott, C. D., et al. 2006, ApJ, 644, 1063

Eichler, D., Livio, M., Piran, T., \& Schramm, D. N. 1989, Nature, 340, 126

Ergma, E. V., \& Tutukov, A. V. 1976, AcA, 26, 69

Faber, J. A., \& Rasio, F. A. 2012, LRR, 15, 8

Fang, J., \& Zhang, L. 2010, A\&A, 515, A20

Fernández, R., \& Metzger, B. D. 2013, MNRAS, 435, 502

Fox, D. B., Frail, D. A., Price, P. A., et al. 2005, Nature, 437, 845

Fried, B. D. 1959, PhFl, 2, 337

Fryer, C., Benz, W., Herant, M., \& Colgate, S. A. 1999, ApJ, 516, 892

Gaensler, B. M., \& Slane, P. O. 2006, ARA\&A, 44, 17

Gao, H., Ding, X., Wu, X. F., Dai, Z. G., \& Zhang, B. 2015, ApJ, 807, 163

Gao, H., Ding, X., Wu, X. F., Zhang, B., \& Dai, Z. G. 2013a, ApJ, 771, 86 
Gao, H., Lei, W. H., Wu, X. F., \& Zhang, B. 2013b, MNRAS, 435, 2520

Gehrels, N., Sarazin, C. L., O’Brien, P. T., et al. 2005, Nature, 437, 851

Geng, J. J., Wu, X. F., Huang, Y. F., \& Yu, Y. B. 2013, ApJ, 779, 28

Giacomazzo, B., \& Perna, R. 2013, ApJL, 771, L26

Giacomazzo, B., Perna, R., Rezzolla, L., et al. 2013, ApJL, 762, L18

Giacomazzo, B., Zrake, J., Duffell, P. C., et al. 2015, ApJ, 809, 39

Gompertz, B. P., O’Brien, P. T., \& Wynn, G. A. 2014, MNRAS, 438, 240

Goriely, S., Bauswein, A., \& Janka, H. T. 2011, ApJL, 738, L32

Gou, L. J., Fox, D. B., \& Mészáros, P. 2007, ApJ, 668, 1083

Greiner, J., Mazzali, P. A., Kann, D. A., et al. 2015, Nature, 523, 189

Harrison, F. A., Craig, W. W., Christensen, F. E., et al. 2013, ApJ, 770, 103

Harrison, F. A., Yost, S. A., Sari, R., et al. 2001, ApJ, 559, 123

Harry, G. M. LIGO Scientific Collaboration, 2010, CQGra, 27, 084006

Hester, J. J. 2008, ARA\&A, 46, 127

Hoshino, M., \& Lyubarsky, Y. 2012, SSRv, 173, 521

Hotokezaka, K., Kiuchi, K., Kyutoku, K., et al. 2013, PhRvD, 87, 024001

Inserra, C., Smartt, S. J., Jerkstrand, A., et al. 2013, ApJ, 770, 128

Just, O., Bauswein, A., Pulpillo, R. A., Goriely, S., \& Janka, H.-T. 2015, MNRAS, 448, 541

Kargaltsev, O., Cerutti, B., Lyubarsky, Y., \& Striani, E. 2015, SSRv, 191, 391

Kasen, D., Badnell, N. R., \& Barnes, J. 2013, ApJ, 774, 25

Kasen, D., \& Bildsten, L. 2010, ApJ, 717, 245

Kasen, D., Fernández, \& Metzger, B. D. 2015, MNRAS, 450, 1777

Kawanaka, N., Mineshige, S., \& Piran, T. 2013, ApJL, 777, L15

Kennel, C. F., \& Coroniti, F. V. 1984a, ApJ, 283, 694 
Kennel, C. F., \& Coroniti, F. V. 1984b, ApJ, 283, 710

Kirk, J. G., \& Skjæaasen, O. 2003, ApJ, 591, 366

Kisaka, S., Ioka, K., \& Nakar, E. 2016, ApJ, 818, 104

Kisaka, S., Ioka, K., \& Takami, H. 2015, ApJ, 802, 119

Kohri, K., \& Mineshige, S. 2002, ApJ, 577, 311

Komissarov, S. S. 2013, MNRAS, 428, 2459

Kulkarni, S. R. 2005, arXiv:astro-ph/0510256

Lee, W. H., Ramirez-Ruiz, E., \& López-Cámara, D. 2009, ApJL, 699, L93

Li, L. X., \& Paczyński, B. 1998, ApJL, 507, L59

Li, S. Z., \& Yu, Y. W. 2016, ApJ, 819, 120

Lippuner, J., \& Roberts, L. F. 2015, ApJ, 815, 82

Lithwick, Y., \& Sari, R. 2001, ApJ, 555, 540

Liu, L. D., Wang, L. J., \& Dai, Z. G. 2016, submitted

Liu, R. Y., Wang, X. Y., \& Wu, X. F. 2013, ApJL, 773, L20

Liu, T., Gu, W. M., Kawanaka, N., \& Li, A. 2015, ApJ, 805, 37

Liu, T., Gu, W. M., Xue, L., \& Lu, J. F. 2007, ApJ, 661, 1025

Lyubarsky, Y. E. 2003, MNRAS, 345, 153

Lyubarsky, Y. 2005, AdSpR, 35, 1112

Lyubarsky, Y. 2010a, ApJL, 725, L234

Lyubarsky, Y. E. 2010b, MNRAS, 402, 353

Lyubarsky, Y., \& Kirk, J. G. 2001, ApJ, 547, 437

Lyubarsky, Y., \& Liverts, M. 2008, ApJ, 682, 1436

Medvedev, M. V., \& Loeb, A. 1999, ApJ, 526, 697

Metzger, B. D. 2012, MNRAS, 419, 827 
Metzger, B. D., \& Berger, E. 2012, ApJ, 746, 48

Metzger, B. D., \& Fernández, R. 2014, MNRAS, 441, 3444

Metzger, B. D., Martínez-Pinedo, G., Darbha, S., et al. 2010, MNRAS, 406, 2650

Metzger, B. D., \& Piro, A. L. 2014, MNRAS, 439, 3916

Metzger, B. D., Piro, A. L., \& Quataert, E. 2008, MNRAS, 390, 781

Metzger, B. D., Piro, A. L., \& Quataert, E. 2009, MNRAS, 396, 304

Michel, F. C. 1973, ApJL, 180, L133

Michel, F. C. 1982, RvMP, 54, 1

Michel, F. C. 1994, ApJ, 431, 397

Michel, F. C., \& Li, H. 1999, PhR, 318, 227

Mizuno, Y., Lyubarsky, Y., Nishikawa, K. I., \& Hardee, P. E. 2011, ApJ, 728, 90

Nakar, E., Ando, S., \& Sari, R. 2009, ApJ, 703, 675

Nakar, E., \& Piran, T. 2011, Nature, 478, 82

Narayan, R., Piran, T., \& Kumar, P. 2001, ApJ, 557, 949

Nicholl, M., Smartt, S. J., Jerkstrand, A., et al. 2014, MNRAS, 444, 2096

Nomoto, K. 1982, ApJ, 253, 798

Nomoto, K., \& Kondo, Y. 1991, ApJL, 367, L19

Nomoto, K., Thielemann, F. K., \& Yokoi, K. 1984, ApJ, 286, 644

Olmi, B., Del Zanna, L., Amato, E., Bandiera, R., \& Bucciantini, N. 2014, MNRAS, 438, 1518

Paczyński, B. 1986, ApJL, 308, L43

Palenzuela, C., Lehner, L., Ponce, M., et al. 2013, PhRvL, 111, 061105

Panaitescu, A., \& Kumar, P. 2000, ApJ, 543, 66

Panaitescu, A., \& Mészáros, P. 1998, ApJ, 501, 772 
Papadopoulos, A., D’Andrea, C. B., Sullivan, M., et al. 2015, MNRAS, 449, 1215

Pétri, J., \& Lyubarsky, Y. 2007, A\&A, 473, 683

Pétri, J., \& Lyubarsky, Y. 2008, IJMPD, 17, 1961

Piran, T., Nakar, E., \& Rosswog, S. 2013, MNRAS, 430, 2121

Popham, R., Woosley, S. E., \& Fryer, C. 1999, ApJ, 518, 356

Porth, O., Komissarov, S. S., \& Keppens, R. 2014, MNRAS, 438, 278

Potekhin, A. Y., De Luca, A., \& Pons, J. A. 2015, SSRv, 191, 171

Rees, M. J., \& Gunn, J. E. 1974, MNRAS, 167, 1

Rezzolla, L., Baiotti, L., Giacomazzo, B., et al. 2010, CQGra, 27, 114105

Rezzolla, L., Giacomazzo, B., Baiotti, L., et al. 2011, ApJL, 732, L6

Roberts, L. F., Kasen, D., Lee, W. H., \& Ramirez-Ruiz, E. 2011, ApJL, 736, L21

Rosswog, S. 2005, ApJ, 634, 1202

Rosswog, S., Korobkin, O., Arcones, A., Thielemann, F. K., \& Piran, T. 2014, MNRAS, 439,744

Rosswog, S., Piran, T., \& Nakar, E. 2013, MNRAS, 430, 2585

Rowlinson, A., O'Brien, P. T., Metzger, B. D., et al. 2013, MNRAS, 430, 1061

Rowlinson, A., O'Brien, P. T., Tanvir, N. R., et al. 2010, MNRAS, 409, 531

Sari, R, \& Esin, A. A. 2001, ApJ, 548, 787

Sari, R., Piran, T., \& Narayan, R. 1998, ApJL, 497, L17

Siegel, D. M., \& Ciolfi, R. 2016a, ApJ, 819, 14

Siegel, D. M., \& Ciolfi, R. 2016b, ApJ, 819, 15

Sironi, L., \& Spitkovsky, A. 2009, ApJL, 707, L92

Sironi, L., \& Spitkovsky, A. 2011a, ApJ, 726, 75

Sironi, L., \& Spitkovsky, A. 2011b, ApJ, 741, 39 
Soderberg, A. M., Berger, E., Kasliwal, M., et al. 2006, ApJ, 650, 261

Somiya, K. (The KAGRA Collaboration) 2012, CQGra, 29, 124007

Song, C. Y., Liu, T., Gu, W. M., Tian, J. X. 2016, MNRAS, 458, 1921

Svensson, R. 1987, MNRAS, 227, 403

Takami, H., Nozawa, T., \& Ioka, K. 2014, ApJL, 789, L6

Tanaka, S. J., \& Takahara, F. 2011, ApJ, 741, 40

Tanvir, N. R., Levan, A. J., Fruchter, A. S., et al. 2013, Nature, 500, 547

Tchekhovskoy, A., Spitkovsky, A., Li, J. G. 2013, MNRAS, 435, L1

Volpi, D., Del Zanna, L., Amato, E., \& Bucciantini, N. 2008, A\&A, 485, 337

Wang, F. Y., \& Dai, Z. G. 2013a, NatPh, 9, 465

Wang, K., \& Dai, Z. G. 2013b, ApJ, 772, 152

Wang, L. J., \& Dai, Z. G. 2013c, ApJL, 774, L33

Wang, L. J., Dai, Z. G., \& Yu, Y. W. 2015a, ApJ, 800, 79

Wang, L. J., Wang, S. Q., Dai, Z. G., et al. 2016, ApJ accepted, arXiv:1602.06190

Wang, S. Q., Liu, L. D., Dai, Z. G., Wang, L. J., \& Wu, X. F. 2015b, arXiv:1509.05543

Wang, S. Q., Wang, L. J., Dai, Z. G., \& Wu, X. F. 2015c, ApJ, 799, 107

Wang, S. Q., Wang, L. J., Dai, Z. G., \& Wu, X. F. 2015d, ApJ, 807, 147

Wang, X. Y., Dai, Z. G., \& Lu, T. 2001a, ApJL, 546, L33

Wang, X. Y., Dai, Z. G., \& Lu, T. 2001b, ApJ, 556, 1010

Wang, X. Y., He, H. N., Li, Z., Wu, X. F., \& Dai, Z. G. 2010, ApJ, 712, 1232

Wei, D. M., \& Lu, T. 1998, ApJ, 505, 252

Weibel, E. S. 1959, PhRvL, 2, 83

Woosley, S. E. 2010, ApJL, 719, L204

Wu, X. F., Gao, H., Ding, X., Zhang, B., Dai, Z. G., Wei, J. Y. 2014, ApJL, 781, L10 
Wu, X. F., Hou, S. J., \& Lei, W. H. 2013, ApJL, 767, L36

Xue, L., Liu, T., Gu, W. M., \& Lu, J. F. 2013, ApJS, 207, 23

Yu, Y. B., Wu, X. F., Huang, Y. F., et al. 2015a, MNRAS, 446, 3642

Yu, Y. W., \& Dai, Z. G. 2007, A\&A, 470, 119

Yu, Y. W., Li, S. Z., \& Dai, Z. G. 2015b, ApJL, 806, L6

Yu, Y. W., Zhang, B., \& Gao, H. 2013, ApJL, 776, L40

Zhang, B. 2013, ApJL, 763, L22

Zhang, B., \& Kobayashi, S. 2005, ApJ, 628, 315

Zhang, B., \& Mészáros, P. 2001, ApJL, 552, L35

Zhang, D., \& Dai, Z. G. 2008, ApJ, 683, 329

Zhang, D., \& Dai, Z. G. 2009, ApJ, 703, 461

Zhang, D., \& Dai, Z. G. 2010, ApJ, 718, 841 
Table 1: Analytical temporal scaling indices of various parameters of the reverse shock IC emission.

\begin{tabular}{|c|c|c|c|c|c|c|c|}
\hline & $\nu_{c}$ & $\nu_{c a}^{\mathrm{IC}}$ & 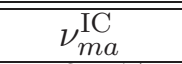 & $\nu_{c c}^{\mathrm{IC}}$ & $\nu_{m c}^{\mathrm{IC}}$ & 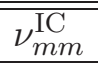 & $\bar{~} F_{\nu, \max }^{\mathrm{IC}}$ \\
\hline$t<T_{N 1}$ & $-\frac{3}{2}$ & $-\frac{3 p+14}{2(p+4)}$ & $-\frac{3 p+14}{2(p+4)}$ & $-\frac{3}{2}$ & $-\frac{3}{2}$ & $-\frac{3}{2}$ & $-\frac{5}{2}$ \\
\hline$T_{N 1}<t<T_{\mathrm{ct}}$ & -3 & $-\frac{3 p+14}{p+4}$ & $-\frac{5 p+22}{p+4}$ & -3 & -5 & -7 & -7 \\
\hline$T_{\mathrm{ct}}<t<T_{a c}$ & 9 & $12-\frac{3 p+2}{p+4}$ & $-\frac{5(p+2)}{p+4}$ & 21 & 7 & -7 & -7 \\
\hline$T_{a c}<t<T_{m c}$ & 9 & $\frac{6(8-3 p)}{5}$ & $-\frac{2(9 p+11)}{5}$ & 21 & 7 & -7 & -7 \\
\hline$T_{m c}<t<T_{\mathrm{dec}}$ & $\frac{8+5 p}{4-p}$ & $\frac{2(p+10)}{4-p}-\frac{13}{5}$ & $-\frac{23}{5}$ & $\frac{7(p+4)}{4-p}$ & $\frac{7 p}{4-p}$ & -7 & -7 \\
\hline$T_{\mathrm{dec}}<t<T_{\mathrm{sd}}$ & $-\frac{2}{4-p}$ & $-\left(\frac{p}{2(4-p)}+\frac{3}{5}\right)$ & $-\frac{1}{10}$ & $-\frac{p+4}{2(4-p)}$ & $-\frac{p}{2(4-p)}$ & $\frac{1}{2}$ & $\frac{1}{2}$ \\
\hline$T_{\mathrm{sd}}<t<T_{N 2}$ & $-\left(\frac{1}{4-p}+\frac{9}{16}\right)$ & $-\left(\frac{1}{4-p}+\frac{3}{4}\right)$ & $-\frac{3}{4}$ & $-\left(\frac{2}{4-p}+\frac{9}{16}\right)$ & $-\left(\frac{1}{4-p}+\frac{9}{16}\right)$ & $-\frac{9}{16}$ & $-\frac{17}{16}$ \\
\hline$T_{N 2}<t$ & $-\frac{3}{5}$ & $-\frac{18}{25}$ & $-\frac{18}{25}$ & $-\frac{3}{5}$ & $-\frac{3}{5}$ & $-\frac{3}{5}$ & $-\frac{7}{5}$ \\
\hline
\end{tabular}

Table 2: Analytical temporal scaling indices of IC flux density of the reverse shock. We have $\nu_{c}<\nu_{a}<\nu_{m}$ if $t<T_{a c}, \nu_{a}<\nu_{c}<\nu_{m}$ if $T_{a c}<t<T_{m c}$, and $\nu_{a}<\nu_{m}<\nu_{c}$ if $t>T_{m c}$.

\begin{tabular}{lcccc}
\hline \hline$\left(\nu_{c}<\nu_{a}<\nu_{m}\right)$ & $\nu<\nu_{c a}^{\mathrm{IC}}$ & \multicolumn{2}{c}{$\nu_{c a}^{\mathrm{IC}}<\nu<\nu_{m m}^{\mathrm{IC}}$} & $\nu_{m m}^{\mathrm{IC}}<\nu$ \\
\hline$t<T_{N 1}$ & $-\frac{2 p+5}{2(p+4)}$ & $-\frac{13}{4}$ & $-\frac{3 p+10}{4}$ \\
$T_{N 1}<t<T_{\mathrm{ct}}$ & $-\frac{4 p+13}{p+4}$ & $-\frac{17}{2}$ & $-\frac{7 p+10}{2}$ \\
$T_{\mathrm{ct}}<t<T_{a c}$ & $-\frac{5(2 p+11)}{p+4}$ & & $\frac{7}{2}$ & $-\frac{7(p-2)}{2}$ \\
\hline$\left(\nu_{a}<\nu_{c}<\nu_{m}\right)$ & $\nu<\nu_{c a}^{\mathrm{IC}}$ & $\nu_{c a}^{\mathrm{IC}}<\nu<\nu_{c c}^{\mathrm{IC}}$ & $\nu_{c c}^{\mathrm{IC}}<\nu<\nu_{m m}^{\mathrm{IC}}$ & $\nu_{m m}^{\mathrm{IC}}<\nu$ \\
\hline$T_{a c}<t<T_{m c}$ & $-\frac{6(17-2 p)}{5}$ & -14 & $\frac{7}{2}$ & $-\frac{7(p-2)}{2}$ \\
\hline$\left(\nu_{a}<\nu_{m}<\nu_{c}\right)$ & $\nu<\nu_{m a}^{\mathrm{IC}}$ & $\nu_{m a}^{\mathrm{IC}}<\nu<\nu_{m m}^{\mathrm{IC}}$ & $\nu_{m m}^{\mathrm{IC}}<\nu<\nu_{c c}^{\mathrm{IC}}$ & $\nu_{c c}^{\mathrm{IC}}<\nu$ \\
\hline$T_{m c}<t<T_{\mathrm{dec}}$ & $-\frac{8}{5}$ & $-\frac{14}{3}$ & $-\frac{7(p+1)}{2}$ & $\frac{7 p(p-2)}{2(4-p)}$ \\
$T_{\mathrm{dec}}<t<T_{\mathrm{sd}}$ & $\frac{2}{5}$ & $\frac{1}{3}$ & $\frac{p+1}{4}$ & $-\frac{p(p-2)}{4(4-p)}$ \\
$T_{\mathrm{sd}}<t<T_{N 2}$ & $-\frac{3}{8}$ & $-\frac{7}{8}$ & $-\frac{9 p+25}{32}$ & $-\left(\frac{9 p+34}{32}+\frac{1}{4-p}\right)$ \\
$T_{N 2}<t$ & $-\frac{18}{25}$ & $-\frac{6}{5}$ & $-\frac{3 p+11}{10}$ & $-\frac{3 p+14}{10}$ \\
\hline
\end{tabular}




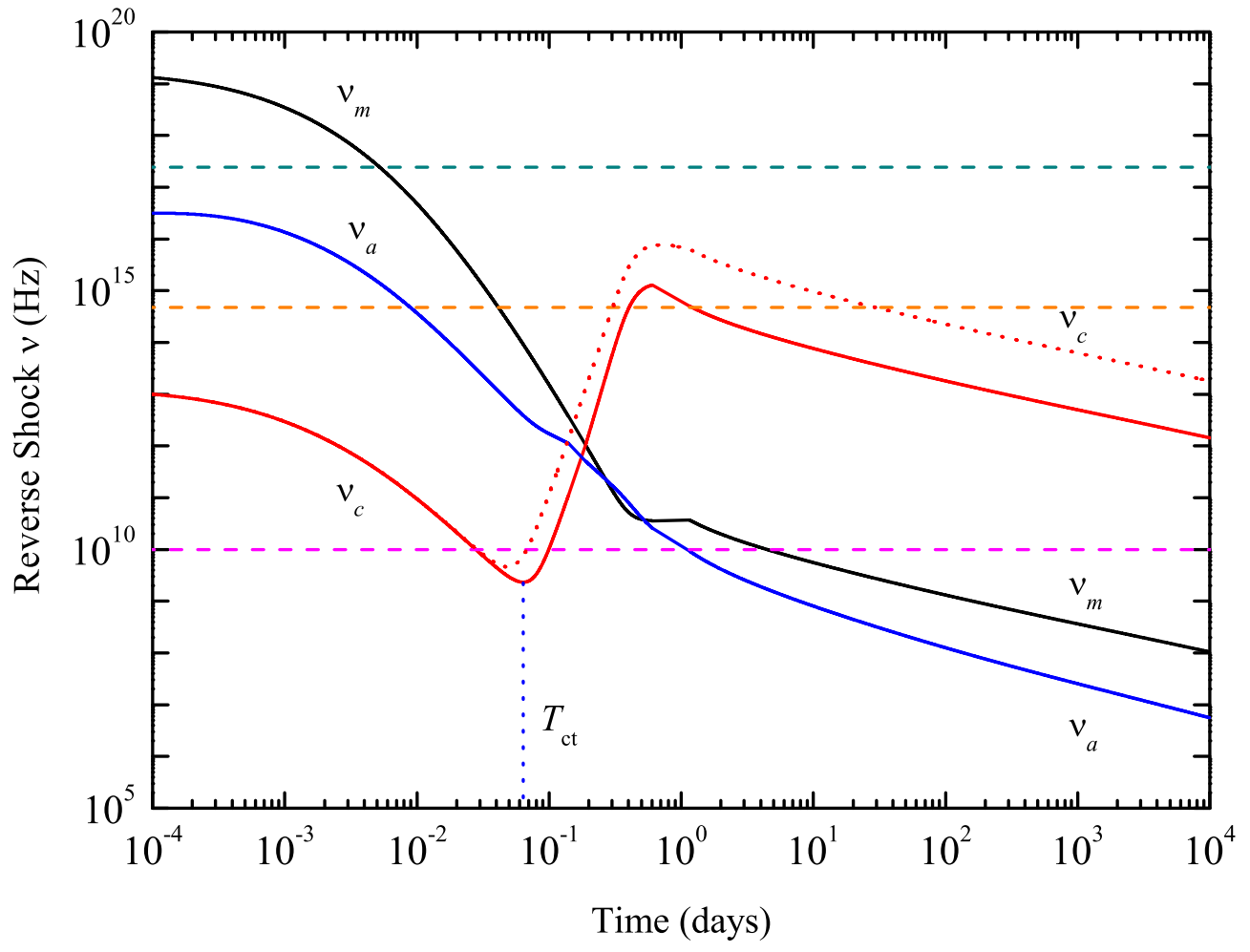

Fig. 1. - The evolution of $\nu_{c}, \nu_{m}$, and $\nu_{a}$ (solid lines) when IC cooling is taken into account. For comparison, $\nu_{c}$ without IC cooling is shown as the dotted line. In this numerical calculation, we adopt Equation (6) to calculate the ejecta dynamics. The three horizontal dashed lines mark the X-ray, optical $(\mathrm{R})$ and radio $(10 \mathrm{GHz})$ bands, respectively. The vertical dotted line indicates the time $T_{\mathrm{ct}}$. 


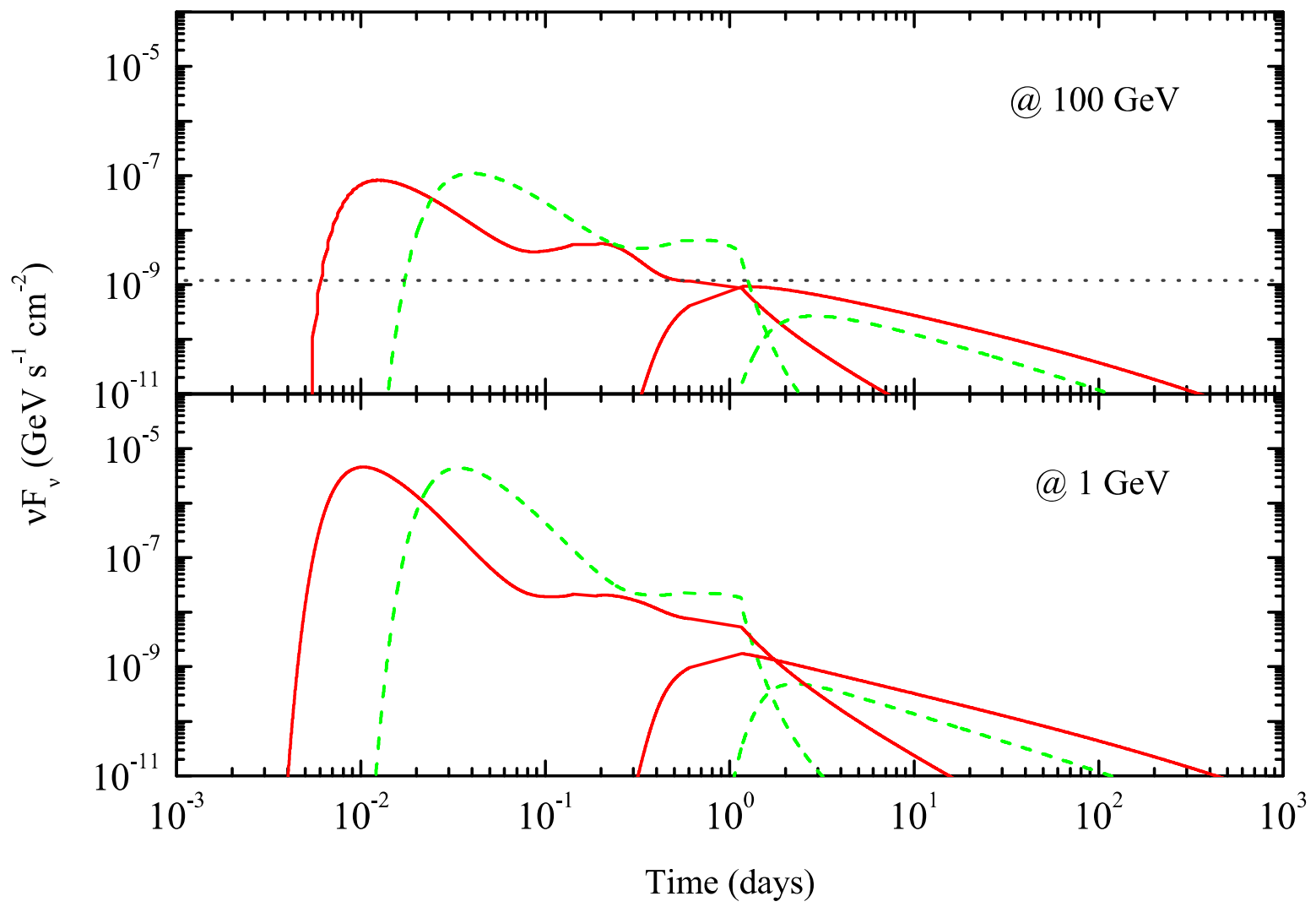

Fig. 2.- Inverse Compton scattered high energy light curves of the reverse shock and forward shock emission at energy bands $1 \mathrm{GeV}$ (lower panel) and $100 \mathrm{GeV}$ (upper panel). The light curves for $M_{\mathrm{ej}}=5 \times 10^{-4} M_{\odot}$ (dashed lines) are compared with the fiducial curves for $M_{\mathrm{ej}}=10^{-4} M_{\odot}$ (solid lines). The short-duration (long-duration) light curves are the reverse (forward) shock emission. Other parameters are $L_{\mathrm{sd}}=10^{47} \mathrm{erg} \mathrm{s}^{-1}, T_{\mathrm{sd}}=10^{5} \mathrm{~s}$, and the luminosity distance $D_{L}=10^{27} \mathrm{~cm}$. The horizontal dotted line in the upper panel marks the detection limit of CTA at $100 \mathrm{GeV}$. In these calculations we neglect $\gamma-\gamma$ collisions, which have no effect on the light curves for the cases of $M_{\mathrm{ej}}=10^{-4} M_{\odot}$ and $M_{\mathrm{ej}}=5 \times 10^{-4} M_{\odot}$. 


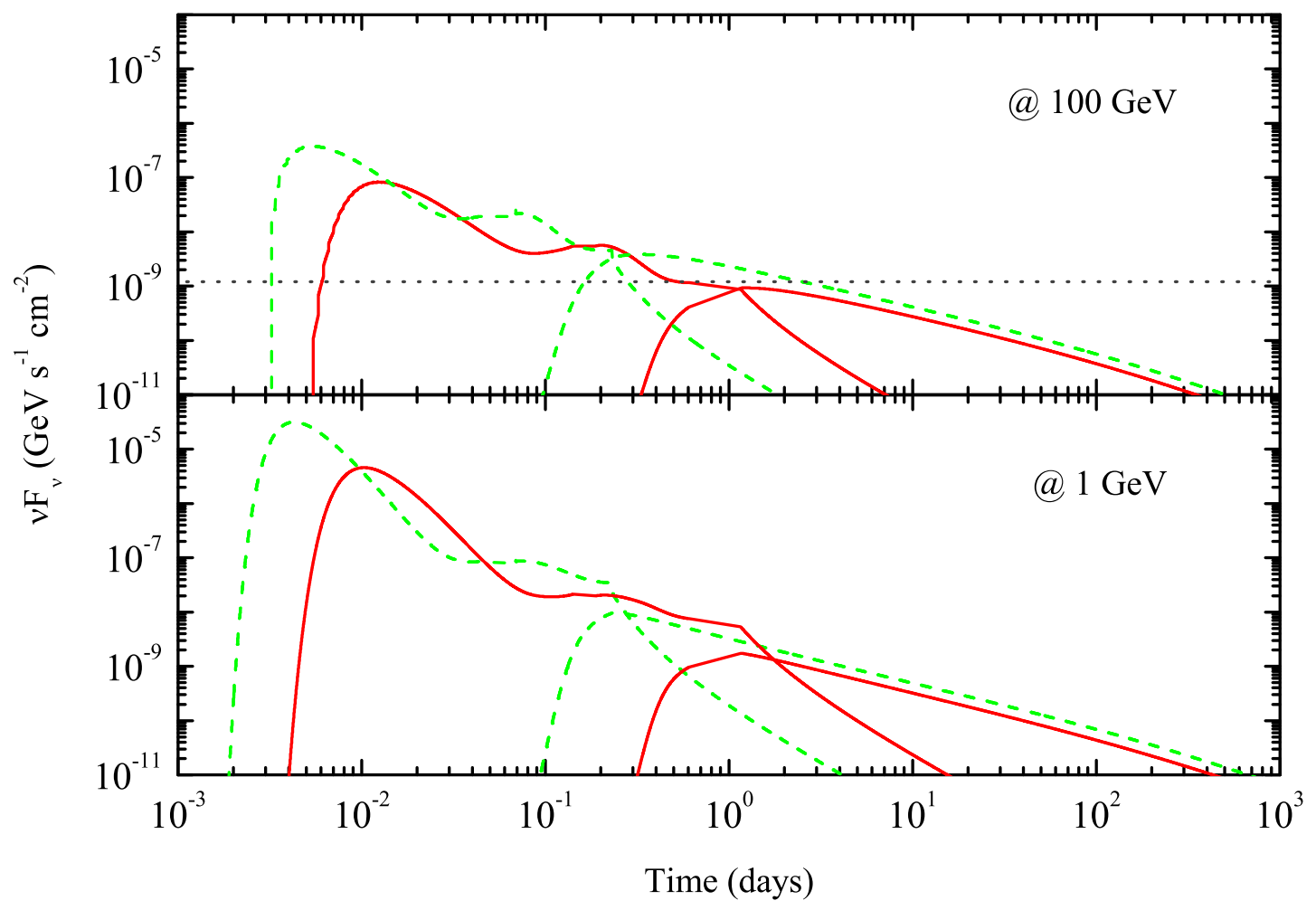

Fig. 3.- The same as Figure 2 but with $L_{\mathrm{sd}}=5 \times 10^{47} \mathrm{erg} \mathrm{s}^{-1}$ for the dashed lines. The total rotational energy of the magnetar is the same as Figure 2 so $T_{\mathrm{sd}}=2 \times 10^{4} \mathrm{~s}$ (dashed lines). The $\gamma-\gamma$ collisions have no influence on the resulting light curves for these cases. 


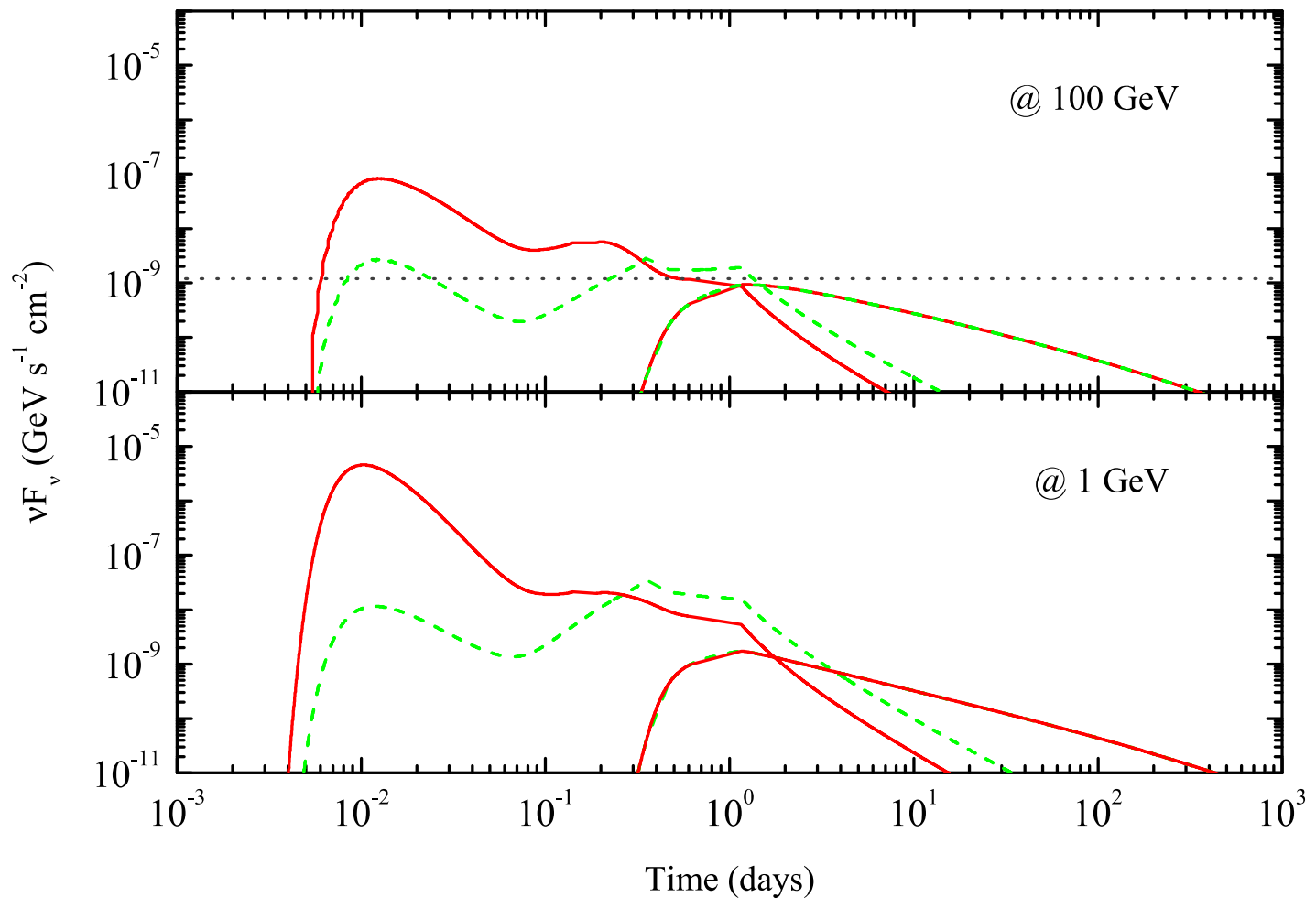

Fig. 4.- The same as Figure 2 but with $\gamma_{4}=10^{6}$ for the dashed lines. The $\gamma-\gamma$ collisions have no influence on the resulting light curves for these cases. 


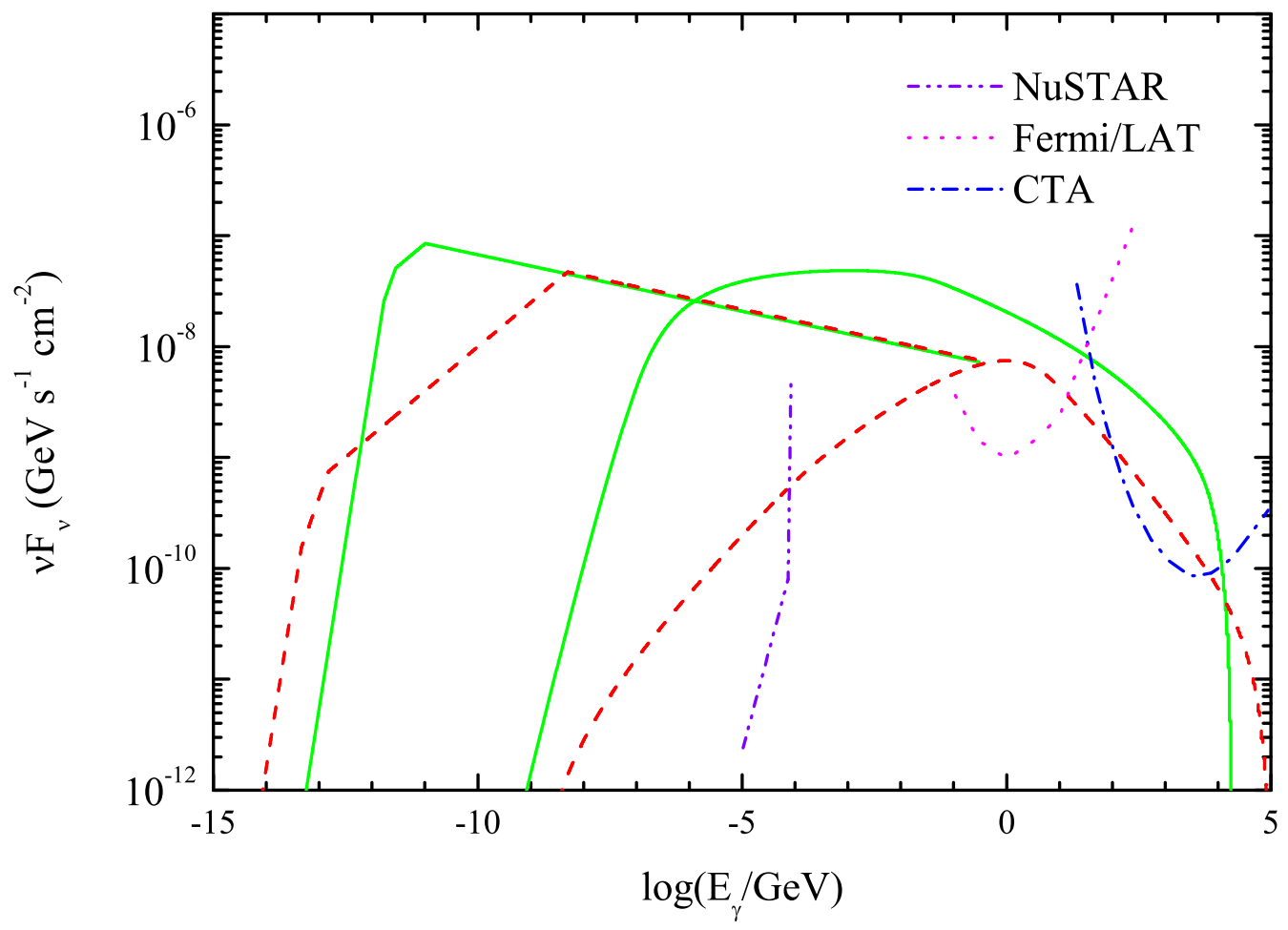

Fig. 5.- Synchrotron (low-frequency components) and IC (high-frequency components) spectra at $t=0.2$ days (solid lines) and $t=1$ days (dashed lines) for the parameters $M_{\mathrm{ej}}=$ $10^{-4} M_{\odot}, L_{\mathrm{sd}}=10^{47} \mathrm{erg} \mathrm{s}^{-1}, T_{\mathrm{sd}}=10^{5} \mathrm{~s}$. Inclusion of $\gamma-\gamma$ collisions would make the early emission (solid lines) at $\gtrsim 10 \mathrm{TeV}$ dimmer. However, given that $10 \mathrm{TeV}$ is close to the IC high-energy cut-off, we expect that the IC spectrum for this case is only slightly modified by including the (thermal) $\gamma-\gamma$ collisions. $\gamma-\gamma$ collisions have no influence for the other case depicted here. 


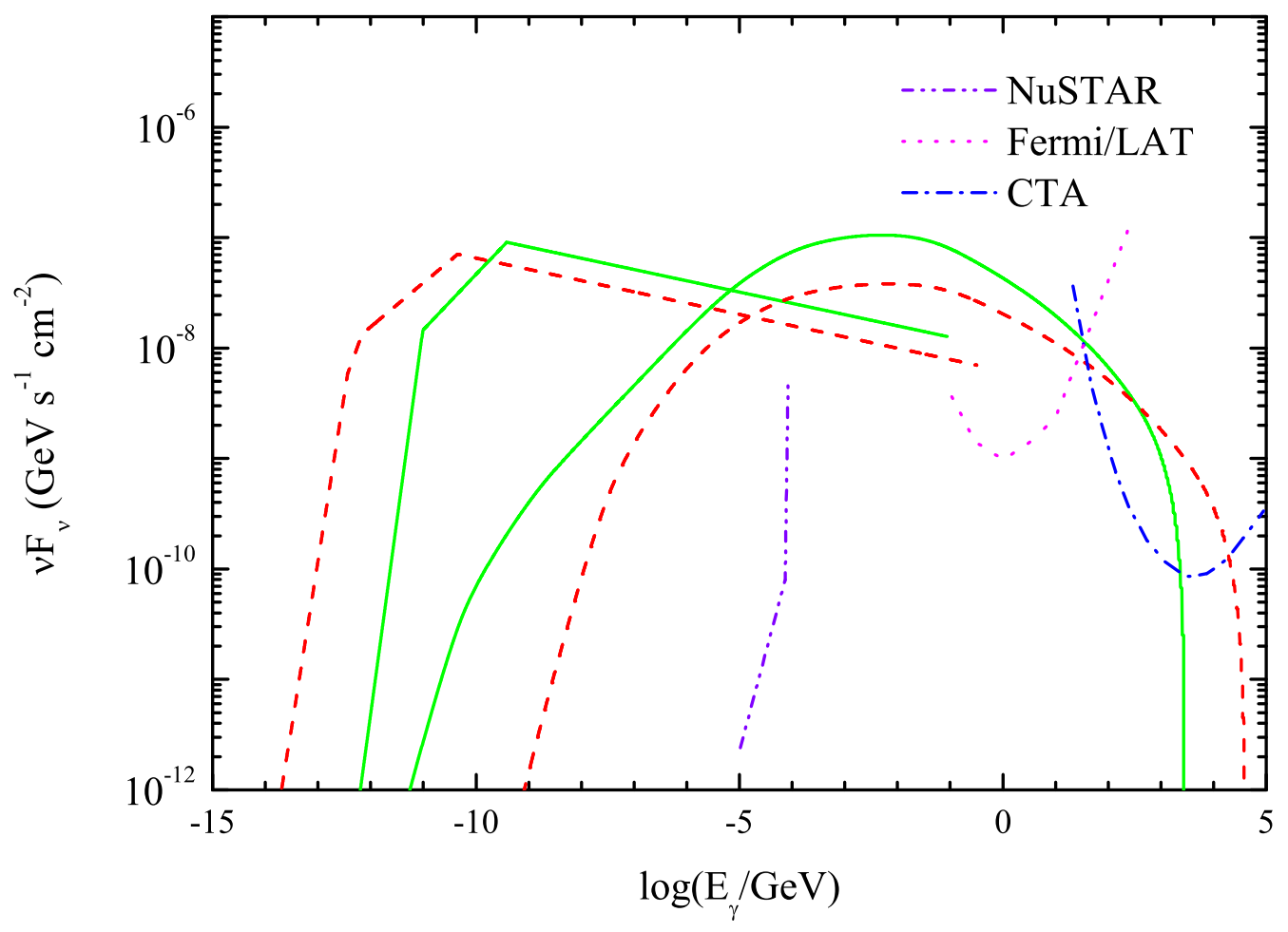

Fig. 6.- The same as Figure 5 but with $M_{\mathrm{ej}}=5 \times 10^{-4} M_{\odot}$, other parameters being the same. $\gamma-\gamma$ collisions have no influence for the cases presented here. 


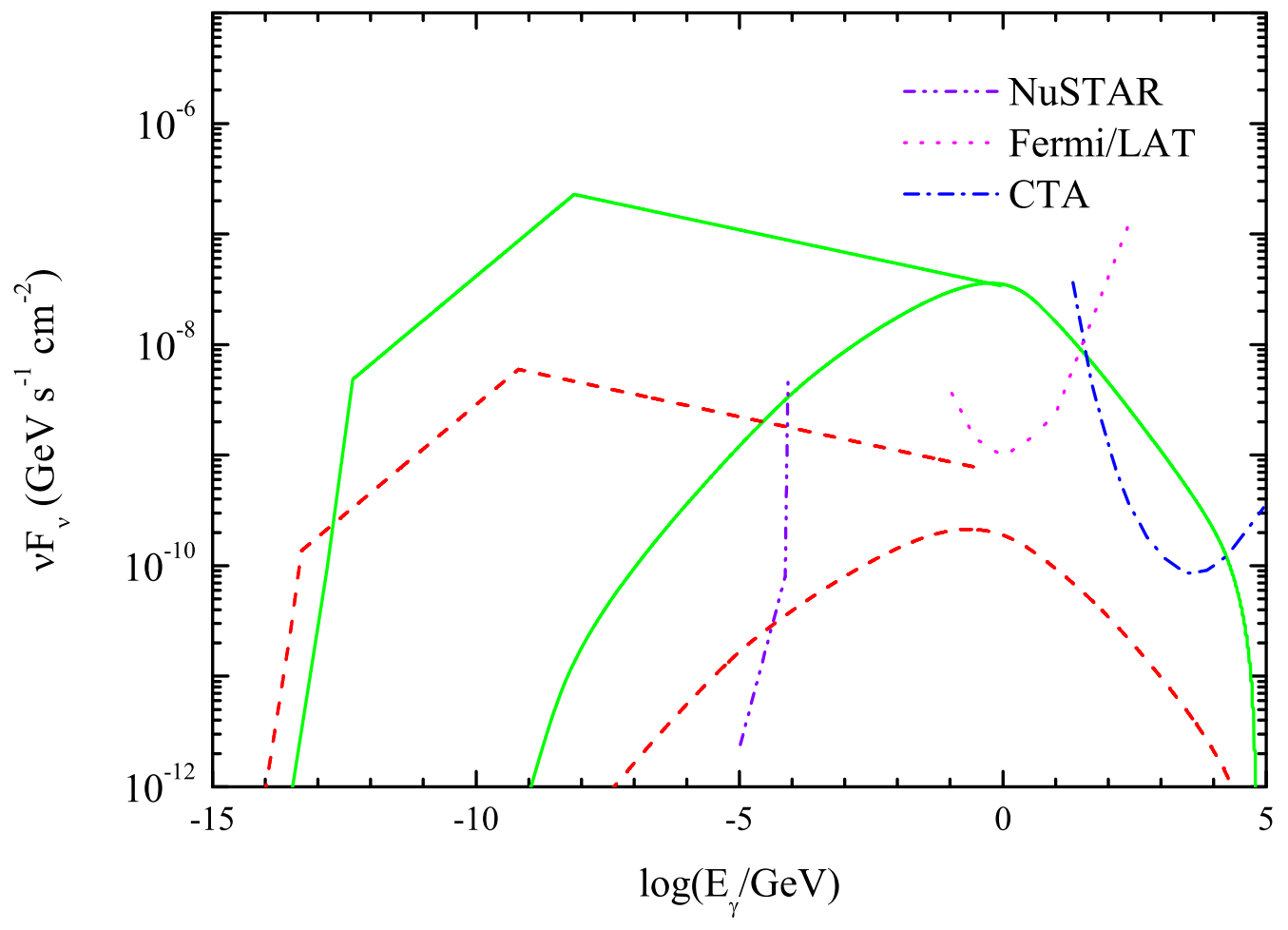

Fig. 7. - The same as Figure 5 but with $L_{\mathrm{sd}}=5 \times 10^{47} \mathrm{erg} \mathrm{s}^{-1}$ and therefore $T_{\mathrm{sd}}=2 \times 10^{4} \mathrm{~s}$, other parameters being the same. $\gamma-\gamma$ collisions have no effect on the spectra for the cases presented here. 


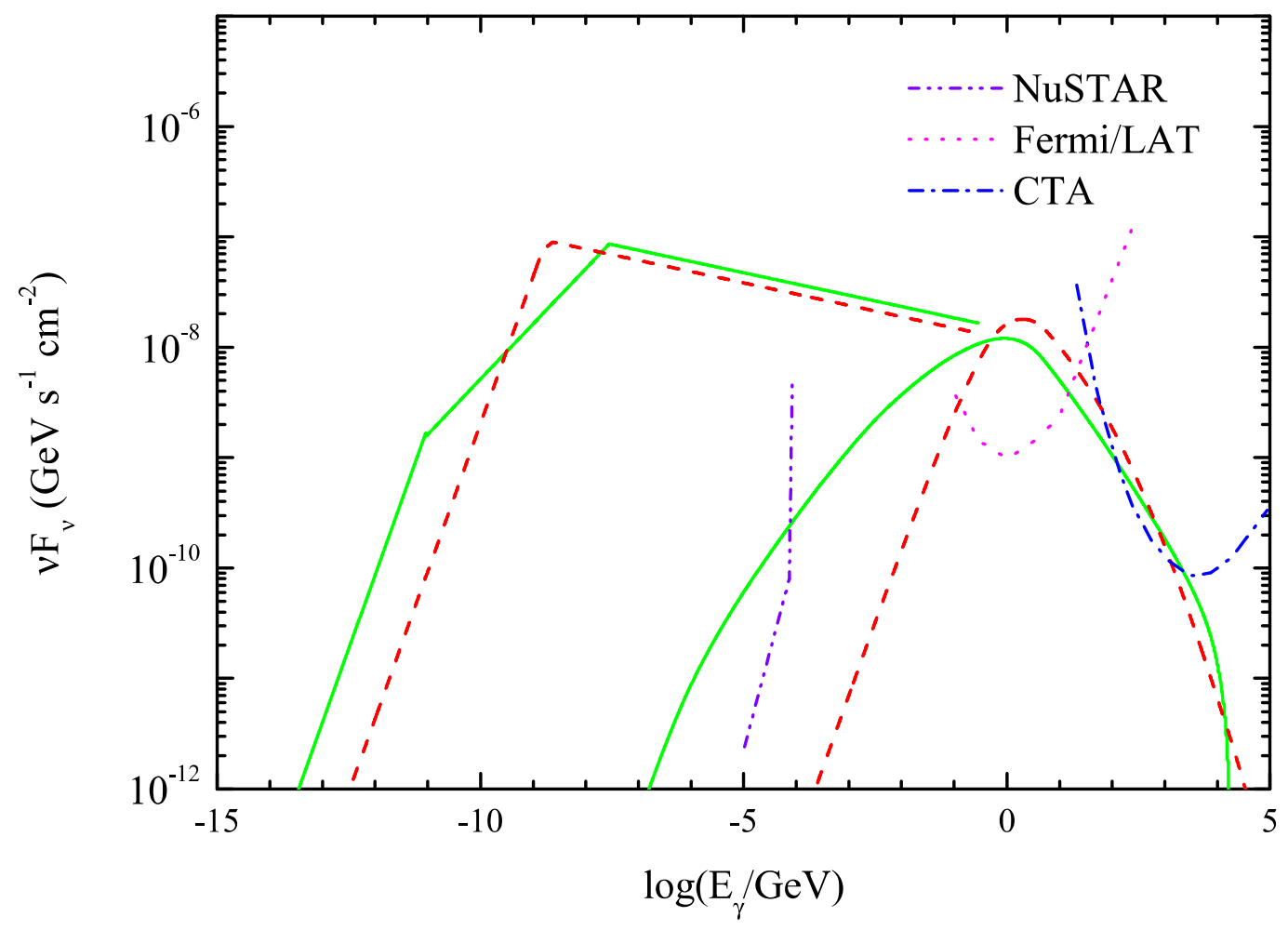

Fig. 8.- The same as Figure 5 but with $\gamma_{4}=10^{6}$, other parameters being the same. $\gamma-\gamma$ collisions have no effect on the spectra for the cases presented here. 